\title{
A Unified Petrov-Galerkin Spectral Method for Fractional PDEs
}

\author{
Mohsen Zayernouri, Mark Ainsworth, and George Em Karniadakis* \\ Division of Applied Mathematics, Brown University, 182 George Street, \\ Providence, RI 02912, USA
}

\begin{abstract}
Existing numerical methods for fractional PDEs suffer from low accuracy and inefficiency in dealing with three-dimensional problems or with longtime integrations. We develop a unified and spectrally accurate PetrovGalerkin (PG) spectral method for a weak formulation of the general linear Fractional Partial Differential Equations (FPDEs) of the form ${ }_{0} \mathcal{D}_{t}^{2 \tau} u+$ $\sum_{j=1}^{d} c_{j}\left[{ }_{a_{j}} \mathcal{D}_{x_{j}}^{2 \mu_{j}} u\right]+\gamma u=f$, where $2 \tau, \mu_{j} \in(0,1)$, in a $(1+d)$-dimensional space-time domain subject to Dirichlet initial and boundary conditions. We perform the stability analysis (in 1-D) and the corresponding convergence study of the scheme (in multi-D). The unified PG spectral method applies to the entire family of linear hyperbolic-, parabolic- and elliptic-like equations. We develop the PG method based on a new spectral theory for fractional Sturm-Liouville problems (FSLPs), recently introduced in [1]. Specifically, we employ the eigenfunctions of the FSLP of first kind (FSLP-I), called Jacobi poly-fractonomials, as temporal/spatial bases. Next, we construct a different space for test functions from poly-fractonomial eigenfunctions of the FSLP of second kind (FSLP-II). Besides the high-order spatial accuracy of the PG method, we demonstrate its efficiency and spectral accuracy in time-integration schemes for solving time-dependent FPDEs as well, rather than employing algebraically accurate traditional methods, especially when $2 \tau=1$. Finally, we formulate a general fast linear solver based on the eigen-pairs of the corresponding temporal and spatial mass matrices with respect to the stiffness matrices, which reduces the computational cost drastically. We demonstrate that this framework can reduce to hyperbolic FPDEs
\end{abstract}

\footnotetext{
${ }^{*}$ Corresponding author: george_karniadakis@brown.edu, Fax: (401)-863-2722

Preprint submitted to CMAME

October 28, 2014
} 
such as time- and space-fractional advection (TSFA), parabolic FPDEs such as time- and space-fractional diffusion (TSFD) model, and elliptic FPDEs such as fractional Helmholtz/Poisson equations with the same ease and cost. Several numerical tests confirm the efficiency and spectral convergence of the unified PG spectral method for the aforementioned families of FPDEs. Moreover, we demonstrate the computational efficiency of the new approach in higher-dimensions e.g., $(1+3),(1+5)$ and $(1+9)$-dimensional problems.

Keywords: Jacobi poly-fractonomial, fractional basis/test functions, unified fast FPDE solver, spectral convergence

\section{Introduction}

The calculus of fractional differentiation and fractional integration generalizes the notion of the standard integer-order calculus to any real-valued order $[2,3]$. Over the last decades, it has been shown that fractional differential operators appear as attractive and potentially powerful modelling tools in many areas such as viscoelastic materials $[4,5]$, porous or fractured media [6], fluid mechanics [7, 8, 9], bioengineering [10], and anomalous diffusion (non-Markovian) processes $[11,12]$. In these applications, fractional partial differential equations (FPDEs) appear of different type such as time- and/or space-fractional diffusion equation of parabolic nature [13], time- and/or space fractional advection, advection-diffusion and Burger's equations of hyperbolic character [14, 15], also elliptic FPDEs as the stationary space-fractional diffusion problems [16].

Existing numerical schemes for FPDEs suffer mainly from low accuracy and computational inefficiency in dealing with three-dimensional problems or with long-time integrations. Recently, a variety of numerical methods, originally developed for integer-order PDEs (see e.g., [17, 18, 19, 20]), have been extended to FPDEs. Such an extension is neither trivial nor straightforward. For instance, there is a lot of work done in developing FiniteDifference Methods (FDM) for FPDEs. Lubich [21, 22] introduced the idea of discretized fractional calculus and later Sanz-Serna [23] presented a firstorder FDM algorithm for partial integro-differential equations. Since then, many works have aimed at improving the convergence rates of FDM schemes e.g., in time to $(\Delta t)^{2-\alpha}$ or $(\Delta t)^{3+\alpha}, \alpha \in(0,1)$ (see e.g.[24, 25, 26, 27]). The implementation of such FDM approaches is relatively easy. However, the bottleneck in the FDM approach is that the convergence is algebraic and the 
accuracy is limited. Moreover, we observe that the heavy cost and memory storage in computing the long-range history in two- and three-dimensional problems makes FDM schemes computationally inefficient. In fact, FDM is essentially a local approach which has been employed to approximate nonlocal fractional derivatives. This fact would suggest that global schemes, such as spectral methods (SM), are more consistent/adapted to the nature of FPDEs.

Sugimoto [15] employed Fourier SM in fractional Burgers' equation, and later Blank [28] adopted a spline-based collocation method for a class of FODEs. This approach was later employed by Rawashdeh [29] for solving fractional integro-differential equations. $\mathrm{Li}$ and $\mathrm{Xu}[30,31]$ developed a space-time spectral method for a time-fractional diffusion equation with spectral convergence, which was based on the early work of Fix and Roop [32]. Later on, Khader [33] proposed a Chebyshev collocation method for a space-fractional diffusion equation; also Piret and Hanert developed a radial basis function method for fractional diffusion equations [34]. Moreover, a Chebyshev spectral method [35], a Legendre spectral method [36], and an adaptive pseudospectral method [37] were proposed for solving fractional boundary value problems. In addition, generalized Laguerre spectral algorithms and Legendre spectral Galerkin method were developed by Baleanu et al. [38] and by Bhrawy and Alghamdi [39] for fractional initial value problems, respectively. The main challenge in these spectral methods is that the corresponding stiffness and mass matrices are non-symmetric, dense and they gradually become ill-conditioned when the fractional order tends to small values. Hence, carrying out long-time and/or adaptive integration using such SM schemes becomes intractable. To this end, $\mathrm{Xu}$ and Hesthaven [40] developed a stable multi-domain spectral penalty method for fractional partial differential equations. In all the aforementioned studies, the standard integer-ordered (polynomial) basis functions have been utilized.

Recently, Zayernouri and Karniadakis [41, 42] developed spectrally accurate Petrov-Galerkin spectral and spectral element methods for non-delay and delay fractional differential equations, where they employed a new family of fractional bases, called Jacobi poly-fractonomials. They introduced these poly-fractonomials as the eigenfunctions of fractional Sturm-Liouville problems in [1], explicitly given as

$$
{ }^{(1)} \mathcal{P}_{n}^{\alpha, \beta, \mu}(\xi)=(1+\xi)^{-\beta+\mu-1} P_{n-1}^{\alpha-\mu+1,-\beta+\mu-1}(\xi), \quad \xi \in[-1,1],
$$

with $\mu \in(0,1),-1 \leq \alpha<2-\mu$, and $-1 \leq \beta<\mu-1$, which are representing 
the eigenfunctions of the singular FSLP of first kind (SFSLP-I), and

$$
{ }^{(2)} \mathcal{P}_{n}^{\alpha, \beta, \mu}(\xi)=(1-\xi)^{-\alpha+\mu-1} P_{n-1}^{-\alpha+\mu-1, \beta-\mu+1}(\xi), \quad \xi \in[-1,1],
$$

where $-1<\alpha<\mu-1$ and $-1<\beta<2-\mu$, and $\mu \in(0,1)$, denoting the eigenfunctions of the singular FSLP of second kind (SFSLP-II). Moreover, Zayernouri and Karniadakis developed a space-time discontinuous PetrovGalerkin (DPG) and a discontinuous Galerkin (DG) method for the hyperbolic time- and space-fractional advection equation in [43]. This approach was shown to be also applicable to problems of integer order time derivatives. In addition, they employed the aforementioned Jacobi poly-fractonomial bases to introduce a new class of fractional Lagrange interpolants for developing an efficient and spectrally accurate Fractional Spectral Collocation Method (FSCM) in [44] for a variety of FODEs and FPDEs including multiterm FPDEs and the nonlinear space-fractional Burgers' equation. Recently, the FSCM scheme has been further generalized to FPDEs of variable order in [45], in where the associate fractional order(s) can vary in the computational domain $\Omega$. However, like all previous spectral methods, applying these schemes to higher-dimensional problems remains a great challenge.

In this paper, we develop a unified and spectrally accurate Petrov-Galerkin (PG) spectral method for the general FPDEs of the following weak form

$$
\left({ }_{0} \mathcal{D}_{t}^{\tau} u,{ }_{t} \mathcal{D}_{T}^{\tau} v\right)_{\Omega}+\sum_{j=1}^{d} c_{j}\left({ }_{a_{j}} \mathcal{D}_{x_{j}}^{\mu_{j}} u,{ }_{x_{j}} \mathcal{D}_{b_{j}}^{\mu_{j}} v\right)_{\Omega}+\gamma(u, v)_{\Omega}=(f, v)_{\Omega},
$$

where $2 \tau, \mu_{j} \in(0,1)$, in a $(1+d)$-dimensional space-time domain subject to Dirichlet initial and boundary conditions. Such a weak form is equivalent to the strong form ${ }_{0} \mathcal{D}_{t}^{2 \tau} u+\sum_{j=1}^{d} c_{x_{j} a_{j}} \mathcal{D}_{x_{j}}^{2 \mu_{j}} u+\gamma u=f$, when $u$ possesses enough smoothness. This method applies equally-well to the entire family of linear fractional hyperbolic, parabolic and elliptic equations with the same ease. The main feature of this PG spectral methods is the global discretization of the temporal term, in addition to the spatial derivatives, rather than utilizing traditional low-order time-integration methods. We essentially develop our PG method based on a new spectral theory for fractional SturmLiouville problems (FSLPs) [1]. Specifically, we employ the eigenfunctions of the FSLP of first kind (FSLP-I), called Jacobi poly-fractonomials, as temporal/spatial bases. Next, we construct a different space for test functions from poly-fractonomial eigenfunctions of the FSLP of second kind (FSLPII). We show that this choice of basis and test functions leads to a stable 
bilinear form; moreover, we perform the corresponding error analysis. In the present method, all the aforementioned matrices are constructed exactly and efficiently. Moreover, we formulate a new general fast linear solver based on the eigen-pairs of the corresponding temporal and spatial mass matrices with respect to the stiffness matrices, which significantly reduces the computational cost in higher-dimensional problems e.g., $(1+3),(1+5)$ and (1+9)-dimensional FPDEs.

The organization of the paper is as follows: in section 2, we introduce the notation and some preliminaries from fractional calculus. In section 3, we present the mathematical formulation of the Petrov-Galerkin spectral method in a $(1+d)$-dimensional hypercube where we define the basis and test function spaces separately. We additionally obtain the general Lyapunov equation, for which we formulate a closed-form solution in terms of the generalised eigensolutions. In section 4, we reduce this general framework to the special well-known (i) hyperbolic, (ii) parabolic FPDEs, and (iii) elliptic FPDEs. We furthermore employ our PG method and the fast solver to even higher dimensional (10-D) problems to demonstrate the robustness and applicability of the scheme. In addition, we introduce our scheme as a spectrally accurate time-integrator method when the FPDE of interest is integer-order in time. We end the paper with a summary and discussion in section 5 . In the Appendix, we present the properties of the stiffness and mass matrices and provide efficient quadrature rules to compute them exactly.

\section{Preliminaries on Fractional Calculus}

We first provide some definitions from fractional calculus. Following [3], for a univariate function $g(x) \in C^{n}[a, b]$, we denote by ${ }_{a} \mathcal{D}_{x}^{\nu} g(x)$ the left-sided Reimann-Liouville fractional derivative of order $\nu$, when $n-1 \leq \nu<n$, defined as

$$
{ }_{a} \mathcal{D}_{x}^{\nu} g(x)=\frac{1}{\Gamma(n-\nu)} \frac{d^{n}}{d x^{n}} \int_{a}^{x} \frac{g(s)}{(x-s)^{\nu+1-n}} d s, \quad x \in[a, b],
$$

where $\Gamma$ represents the Euler gamma function, and as $\nu \rightarrow n$, the global operator ${ }_{a} \mathcal{D}_{x}^{\nu} \rightarrow d^{n} / d x^{n}$, recovering the local $n$-th order derivative with respect to $x$. We also denote by ${ }_{x} \mathcal{D}_{b}^{\nu} g(x)$ the corresponding right-sided ReimannLiouville fractional derivative of order $\nu$, defined as

$$
{ }_{b} \mathcal{D}_{x}^{\nu} g(x)=\frac{1}{\Gamma(n-\nu)}(-1)^{n} \frac{d^{n}}{d x^{n}} \int_{x}^{b} \frac{g(s)}{(s-x)^{\nu+1-n}} d s, \quad x \in[a, b] .
$$


Similarly, as $\nu \rightarrow n$, the right-sided fractional derivative tends to the standard $n$-th local one. The corresponding left- and right-sided fractional derivative of Caputo type can be also defined as (3) and (4), but with the order of integration and differentiation exchanged. However, these two sets of RiemannLiouville and Caputo definitions are closely linked. By virtue of (3) and (4), we can define the corresponding partial fractional-derivative of a bivariate function.

Finally, we recall a useful property of the Riemann-Liouville fractional derivatives. Assume that $0<p<1$ and $0<q<1$ and $g\left(x_{L}\right)=0 x>x_{L}$, then

$$
{ }_{x_{L}} \mathcal{D}_{x}^{p+q} g(x)=\left({ }_{{ }_{x}} \mathcal{D}_{x}^{p}\right)\left({ }_{x_{L}} \mathcal{D}_{x}^{q}\right) g(x)=\left({ }_{x_{L}} \mathcal{D}_{x}^{q}\right)\left({ }_{x_{L}} \mathcal{D}_{x}^{p}\right) g(x) .
$$

\section{Mathematical Formulation of Petrov-Galerkin Spectral Method}

Let $u: \mathbb{R}^{d+1} \rightarrow \mathbb{R}$, for some positive integer $d$. For $u \in U$ (see e.g., (10)), we consider the following general weak form in $\Omega=[0, T] \times\left[a_{1}, b_{1}\right] \times\left[a_{2}, b_{2}\right] \times$ $\cdots \times\left[a_{d}, b_{d}\right]$ as

$$
\left({ }_{0} \mathcal{D}_{t}^{\tau} u,{ }_{t} \mathcal{D}_{T}^{\tau} v\right)_{\Omega}+\sum_{j=1}^{d} c_{j}\left({ }_{a_{j}} \mathcal{D}_{x_{j}}^{\mu_{j}} u,{ }_{x_{j}} \mathcal{D}_{b_{j}}^{\mu_{j}} v\right)_{\Omega}+\gamma(u, v)_{\Omega}=(f, v)_{\Omega}, \quad \forall v \in V,
$$

where $\gamma, c_{j}$ are constant, $2 \tau, \mu_{j} \in(0,1), j=1,2, \cdots, d$, subject to the following homogeneous Dirichlet initial and boundary conditions

$$
\begin{aligned}
&\left.u\right|_{t=0}=0, \quad \tau \in(0,1 / 2), \\
&\left.u\right|_{x_{j}=a_{j}}=0, \quad \mu_{j} \in(0,1 / 2), \quad j=1,2, \cdots, d \\
&\left.u\right|_{x_{j}=a_{j}}=\left.u\right|_{x_{j}=b_{j}}=0, \quad \mu_{j} \in(1 / 2,1), \quad j=1,2, \cdots, d .
\end{aligned}
$$

We note that the variational form (6) is equivalent to the following linear FPDE of order $2 \tau$ in time and $2 \mu_{j}$ in the $j$-th spatial dimension, $j=$ $1,2, \cdots, d$,

$$
\begin{aligned}
{ }_{0} \mathcal{D}_{t}^{2 \tau} u\left(t, x_{1}, x_{2}, \cdots, x_{d}\right) & +\sum_{j=1}^{d} c_{j}\left[{ }_{a_{j}} \mathcal{D}_{x_{j}}^{2 \mu_{j}} u\left(t, x_{1}, x_{2}, \cdots, x_{d}\right)\right] \\
& +\gamma u\left(t, x_{1}, x_{2}, \cdots, x_{d}\right)=f\left(t, x_{1}, x_{2}, \cdots, x_{d}\right),
\end{aligned}
$$

when solution $u$ is smooth enough. 
We define the solution space $U$ as

$U:=\left\{u: \Omega \rightarrow \mathbb{R} \mid u \in C(\Omega),\|u\|_{U}<\infty\right.$, s.t. $\left.\left.u\right|_{t=0}=\left.u\right|_{x_{j}=a_{j}}=\left.u\right|_{x_{j}=b_{j}}=0\right\}$ when $\mu_{j} \in(1 / 2,1)$, in which

$$
\|u\|_{U}=\left\{\left\|{ }_{0} \mathcal{D}_{t}^{\tau} u\right\|^{2}+\sum_{j=1}^{d}\left\|{ }_{a_{j}} \mathcal{D}_{x_{j}}^{\mu_{j}} u\right\|^{2}+\|u\|^{2}\right\}^{1 / 2}
$$

Correspondingly, we define the test space $V$ as

$$
V:=\left\{v: \Omega \rightarrow \mathbb{R} \mid\|v\|_{V}<\infty, \text { s.t. }\left.v\right|_{t=T}=\left.v\right|_{x_{j}=b_{j}}=0\right\}
$$

when $\mu_{j} \in(0,1)$, in which

$$
\|v\|_{V}=\left\{\left\|{ }_{t} \mathcal{D}_{T}^{\tau} v\right\|^{2}+\sum_{j=1}^{d}\left\|{ }_{x_{j}} \mathcal{D}_{b_{j}}^{\mu_{j}} v\right\|^{2}+\|v\|^{2}\right\}^{1 / 2}
$$

where by $[32,46]$, we can show that $U$ and $V$ are Hilbert spaces, moreover, the associated norms $\|\cdot\|_{U}$ and $\|\cdot\|_{V}$ are equivalent. Now, let $a: U \times V \rightarrow \mathbb{R}$ be a bilinear form, defined as

$$
a(u, v)=\left({ }_{0} \mathcal{D}_{t}^{\tau} u,{ }_{t} \mathcal{D}_{T}^{\tau} v\right)_{\Omega}+\sum_{j=1}^{d} c_{j}\left({ }_{a_{j}} \mathcal{D}_{x_{j}}^{\mu_{j}} u,{ }_{x_{j}} \mathcal{D}_{b_{j}}^{\mu_{j}} v\right)_{\Omega}+\gamma(u, v)_{\Omega}
$$

Moreover, let $L \in V^{*}$, the dual space of $V$, be a continuous linear functional defined as

$$
L(v)=(f, v), \quad \forall v \in V .
$$

Now, the problem is to find $u \in U$ such that

$$
a(u, v)=L(v), \quad \forall v \in V .
$$


Next, we define $U_{N} \subset U$ and $V_{N} \subset V$ to be finite dimensional subspaces of $U$ and $V$ with $\operatorname{dim}\left(U_{N}\right)=\operatorname{dim}\left(V_{N}\right)=N$. Now, our PG spectral method reads as: find $u_{N} \in U_{N}$ such that

$$
a\left(u_{N}, v_{N}\right)=L\left(v_{N}\right), \quad \forall v_{N} \in V_{N} .
$$

By representing $u_{N}$ as a linear combination of points/elements in $U_{N}$ i.e., the corresponding $(1+d)$-dimensional space-time basis functions, the finitedimensional problem (17) leads to a linear system known as Lyapunov matrix equation. For instance, if $d=1$, i.e., $1-\mathrm{D}$ in time and 1-D space, we obtain the corresponding Lyapunov equation in the space-time domain $[0, T] \times\left[a_{1}, b_{1}\right]$ as

$$
S_{\tau} \mathcal{U} M_{\mu_{1}}^{T}+M_{\tau} \mathcal{U} S_{\mu_{1}}^{T}=F,
$$

in which $\mathcal{U}$ is the matrix of unknown coefficients, $S_{\tau}$ and $M_{\tau}$ denote, respectively, the temporal stiffness and mass matrices; similarly, $S_{\mu_{1}}$ and $M_{\mu_{1}}$, represent the spatial stiffness and mass matrices, and $\mathrm{F}$ is the corresponding load matrix.

In general, numerical solutions to such a linear system, originating from a fractional differential operator, become excessively expensive since the corresponding mass and stiffness matrices usually turn out to be full and nonsymmetric. Moreover, we note that the size of the above linear system grows as the product of the degrees of freedom in each dimension. To address this problem in this paper, we present a new class of basis and test functions yielding stiffness matrices, which are either diagonal or tridiagonal. Similarly, by introducing proper quadrature rules, we compute exactly the corresponding mass matrices, which are symmetric. Such useful properties allow us to subsequently develop a general fast linear solver for (18) with a substantially reduced computational cost. To this end, we first introduce the corresponding finite-dimensional spaces of basis $U_{N}$ and test functions $V_{N}$ in our PG framework.

\subsection{Space of Basis Functions $\left(U_{N}\right)$}

We develop a PG spectral method for (8), subject to homogeneous Dirichlet initial and boundary conditions. We construct the basis function space as the space of some temporal and spatial functions to globally treat the time-dimension in addition to the spatial-dimensions. To this end, the new eigensolutions, introduced in [1], yield new sets of basis and test functions, 
properly suited for our Petrov-Galerkin framework. We represent the solution in the entire space-time computational domain $\Omega$ in terms of specially chosen basis functions, constructed as the tensor product of the the eigenfunctions in the following manner. Let

$$
{ }^{(1)} \mathcal{P}_{n}{ }^{\mu}(\xi)=(1+\xi)^{\mu} P_{n-1}^{-\mu, \mu}(\xi), \quad n=1,2, \cdots \quad x \in[-1,1],
$$

denote the eigenfunctions of the regular FSLP of first kind (RFSLP-I), corresponding to the case where $\alpha=\beta=-1$. We construct our basis for the spatial discretization using the univariate poly-fractonomials defined by

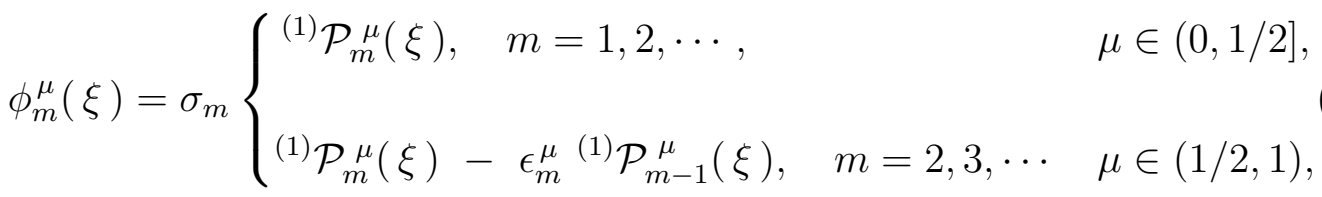

where $\sigma_{m}=2+(-1)^{m}$ and the $\mu$-dependent coefficient $\epsilon_{m_{j}}^{\mu}=(m-1-\mu) /(m-$ $1)$. The definition reflects the fact that if $\mu \leq 1 / 2$ then only one boundary condition needs to be presented, whereas if $\mu>1 / 2$ then two endpoint conditions are prescribed. Naturally, for the temporal basis functions only initial conditions are prescribed and as a consequence the basis functions for the temporal discretization are constructed using the univariate polyfractonomials

$$
\psi_{n}^{\tau}(\eta)=\sigma_{n}{ }^{(1)} \mathcal{P}_{n}^{\tau}(\eta), \quad \tau \in(0,1),
$$

for $n \geq\lceil 2 \tau\rceil$. With these notations established, we define the space-time trial space to be

$U_{N}=\operatorname{span}\left\{\left(\psi_{n}^{\tau} \circ \eta\right)(t) \prod_{j=1}^{d}\left(\phi_{m_{j}}^{\mu_{x_{j}}} \circ \xi_{j}\right)\left(x_{j}\right): n=1, \ldots, \mathcal{N}, m_{j}=\left\lceil\sigma_{j}\right\rceil, \ldots, \mathcal{M}_{j}\right\}$

where $\eta(t)=2 t / T-1$ and $\xi_{j}(s)=2 \frac{s-a_{j}}{b_{j}-a_{j}}-1$. The construction of the univariate functions ensures that $U_{N} \subset U$, since $\phi_{m}^{\mu}(-1)=0$, for all $\mu \in$ $(0,1)$, also $\phi_{m}^{\mu}(1)=0$, for all $\mu \in(1 / 2,1)$. Then, we shall approximate the solution to (8) in terms of a linear combination of elements in $U_{N}$, whose bases satisfy exactly the homogeneous initial and boundary condition in $\Omega$.

\subsection{Space of Test Functions $\left(V_{N}\right)$}

Let the poly-fractonomials

$$
{ }^{(2)} \mathcal{P}_{k}^{\mu}(\xi)=(1-\xi)^{\mu} P_{k-1}^{\mu,-\mu}(\xi), \quad k=1,2, \cdots, \xi \in[-1,1],
$$


denote the eigenfunctions of the regular FSLP of second kind (RFSLP-II), corresponding to the case $\alpha=\beta=-1$ in (2). Next, we construct our spatial test functions using the univariate poly-fractonomials defined by

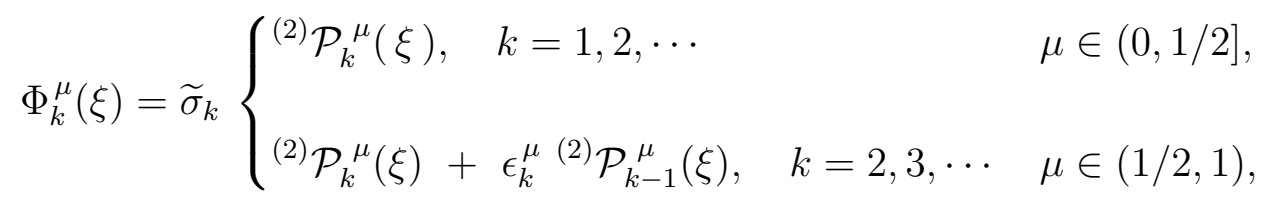

where $\widetilde{\sigma}_{k}=2(-1)^{k}+1$. Next, we define the temporal test functions using the univariate poly-fractonomials

$$
\Psi_{r}^{\tau}(\eta)=\widetilde{\sigma}_{r}{ }^{(2)} \mathcal{P}_{r}^{\tau}(\eta), \quad \tau \in(0,1),
$$

for all $r \geq\lceil 2 \tau\rceil$. With these notations established, we define the space-time test space to be

$$
V_{N}=\operatorname{span}\left\{\left(\Psi_{r}^{\tau} \circ \eta\right)(t) \prod_{j=1}^{d}\left(\Phi_{k_{j}}^{\mu_{j}} \circ \xi_{j}\right)\left(x_{j}\right): r=1, \ldots, \mathcal{N}, k_{j}=\left\lceil\sigma_{j}\right\rceil, \ldots, \mathcal{M}_{j}\right\} .
$$

Having defined the space of trial and test functions, we can now define the corresponding temporal/spatial stiffness and mass matrices.

Remark 3.1. We show later that the choice of $\sigma_{m}$ in (20) and (21), also $\widetilde{\sigma}_{k}$ in (24) and (25), leads to the construction of symmetric spatial/temporal mass and stiffness matrices. We will exploit this property to formulate a general fast linear solver for the resulting linear system.

\subsection{Stability and Convergence Analysis}

The following theorems provide the stability analysis of the scheme when the pair of $U_{N} \subset U$ and $V_{N} \subset V$ are given as in (22) and (26), respectively. We first consider the discrete stability of the method for one-dimensional case.

Theorem 3.2. The Petrov-Gelerkin spectral method for the problem

$$
\begin{aligned}
{ }_{-1} \mathcal{D}_{x}^{2 \mu} u(x) & =f(x), \quad \forall x \in[-1,1], \\
u(-1) & =0, \quad \text { if } 0<\mu<1 / 2, \\
u( \pm 1) & =0, \quad \text { if } 1 / 2<\mu<1
\end{aligned}
$$


is stable, i.e., the discrete inf-sup condition

$$
\sup _{v_{N} \in V_{N}} \frac{a\left(u_{N}, v_{N}\right)}{\left\|v_{N}\right\|_{V}} \geq \beta\left\|u_{N}\right\|_{U}, \quad \forall u_{N} \in U_{N} \subset U,
$$

holds with $\beta=1$.

Proof. We note that in the absence of the time-derivative and since $\gamma=0$, the corresponding norm defined on $U$ (see Eq. 11) just reduces to $\|u\|_{U}=$ $\left\|_{-1} \mathcal{D}_{x}^{\mu} u\right\|$. While $\left\|_{-1} \mathcal{D}_{x}^{\mu} u\right\|$ has been traditionally treated as a semi-norm in the literature (e.g., see $[47,30]$ ), one can easily show that it satisfies all the properties of a norm since the Riemann-Liouville fractional derivative of a constant is non-zero. Correspondingly, in this one-dimensional setting, $\|v\|_{V}=\left\|{ }_{x} \mathcal{D}_{1}^{\mu} v\right\|$.

Case I) $0<\mu<1 / 2$ : we represent $u_{N}$ as

$$
u_{N}(x)=\sum_{n=1}^{N} \hat{u}_{n}(1+x)^{\mu} P_{n-1}^{-\mu, \mu}(x),
$$

and choose $v_{N}$ to be the following linear combination of elements in $V_{N}$ as

$$
v_{N}(x)=\sum_{k=1}^{N} \hat{u}_{k}(1-x)^{\mu} P_{k-1}^{\mu,-\mu}(x),
$$

in which we employ the same coefficients $\hat{u}_{k}$ as in (29). Hence, we obtain

$$
\begin{aligned}
& a\left(u_{N}, v_{N}\right)=\int_{-1}^{1}{ }_{-1} \mathcal{D}_{x}^{\mu} u_{N}{ }_{x} \mathcal{D}_{1}^{\mu} v_{N} d t \\
= & \sum_{n=1}^{N} \hat{u}_{n} \sum_{k=1}^{N} \hat{u}_{k} \int_{-1}^{1}{ }_{-1} \mathcal{D}_{x}^{\mu}\left[(1+x)^{\mu} P_{n-1}^{-\mu, \mu}(x)\right],{ }_{x} \mathcal{D}_{1}^{\mu}\left[(1-x)^{\mu} P_{k-1}^{\mu,-\mu}(x)\right] d t \\
= & \sum_{n=1}^{N} \hat{u}_{n} \frac{\Gamma(n+\mu)}{\Gamma(n)} \sum_{k=1}^{N} \hat{u}_{k} \frac{\Gamma(k+\mu)}{\Gamma(k)} \int_{-1}^{1} P_{n-1}(x) P_{k-1}(x) d t \\
= & \sum_{n=1}^{N} \hat{u}_{n}^{2}\left(\frac{\Gamma(n+\mu)}{\Gamma(n)}\right)^{2} \frac{2 n+1}{2} \\
= & \left\|_{x} \mathcal{D}_{1}^{\mu} v_{N}\right\|_{L^{2}([-1,1])}^{2}=\left\|v_{N}\right\|_{V}^{2},
\end{aligned}
$$




$$
\sup _{v_{N} \in V_{N}} \frac{a\left(u_{N}, v_{N}\right)}{\left\|v_{N}\right\|_{V}}=\left\|u_{N}\right\|_{U}, \quad \forall u_{N} \in U_{N}
$$

which means that the stability is guaranteed for $\beta=1$.

Case II) $1 / 2<\mu<1$ : we expand $u_{N}$ this time as

$$
u_{N}(x)=\sum_{n=1}^{N} \hat{u}_{n}\left[(1+x)^{\mu} P_{n-1}^{-\mu, \mu}(x)-\epsilon_{n}^{\mu}(1+x)^{\mu} P_{n-2}^{-\mu, \mu}(x)\right],
$$

and choose $v_{N}$ to be the following linear combination of elements in $V_{N}$ as

$$
v_{N}(x)=\sum_{k=1}^{N} \hat{u}_{k}\left[(1-x)^{\mu} P_{k-1}^{\mu,-\mu}(x)+\epsilon_{k}^{\mu}(1-x)^{\mu} P_{k-2}^{\mu,-\mu}(x)\right],
$$

where the coefficients $\hat{u}_{k}$ are the same as the ones in (33). Hence, it is easy to again show that

$$
\begin{aligned}
a\left(u_{N}, v_{N}\right) & =\sum_{n=1}^{N} \hat{u}_{n}^{2}\left(\frac{\Gamma(n+\mu)}{\Gamma(n)}\right)^{2} \frac{2 n+1}{2}\left(1-\epsilon_{n}^{\mu} \mathbb{I}_{\{1 \leq n \leq N-1\}}\right) \\
& =\left\|_{x} \mathcal{D}_{1}^{\mu} v_{N}\right\|_{L^{2}([-1,1])}^{2}=\left\|v_{N}\right\|_{V}^{2} .
\end{aligned}
$$

Remark 3.3. We performed the discrete stability analysis for the 1-D case. The multi-D case is more involved and we will address it in a separate paper in future.

Theorem 3.4. (Projection Error) In the weak form (17), let $\left\|_{0} \mathcal{D}_{t}^{r+\tau} u\right\|_{L^{2}(\Omega)}<$ $\infty$ and $\left\|_{-1} \mathcal{D}_{x_{j}}^{r+\mu_{j}} u\right\|_{L^{2}(\Omega)}<\infty$ for all $j=1,2, \cdots, d$, for some integer $r \geq 1$. Moreover, let $u_{N}$ denote the projection of the exact solution u.Then,

$$
\left\|u-u_{N}\right\|_{U}^{2} \leq C N^{-2 r}\left(\left\|_{0} \mathcal{D}_{t}^{r+\tau} u\right\|_{L^{2}(\Omega)}^{2}+\sum_{j=1}^{d}\left\|{ }_{-1} \mathcal{D}_{x_{j}}^{r+\mu_{j}} u\right\|_{L^{2}(\Omega)}^{2}\right) .
$$

Proof. We first consider the one-dimensional problem (27). We expand the exact solution $u$, when $2 \mu \in(0,1)$, in terms of the following infinite series of Jacobi poly-fractonomials

$$
u(x)=\sum_{n=1}^{\infty} \hat{u}_{n}(1+x)^{\mu} P_{n-1}^{-\mu, \mu}(x) .
$$


Here, we would like to bound $\left\|u-u_{N}\right\|_{U}$ in terms of higher-order derivative We first note that

$$
{ }_{-1} \mathcal{D}_{x}^{r+\mu} u(x)=\frac{d^{r}}{d x^{r}}\left[{ }_{-1} \mathcal{D}_{x}^{\mu} u(x)\right]=\sum_{n=1}^{\infty} \hat{u}_{n} \frac{\Gamma(n+\mu)}{\Gamma(n)} \frac{d^{r}}{d x^{r}}\left[P_{n-1}(x)\right],
$$

where

$$
\frac{d^{r}}{d x^{r}}\left[P_{n-1}(x)\right]= \begin{cases}\frac{(n-1+r) !}{2 r(n-1) !} P_{n-1-r}^{r, r}(x), & r \leq n \\ 0, & r>n\end{cases}
$$

Hence,

$$
{ }_{-1} \mathcal{D}_{x}^{r+\mu} u(x)=\sum_{n=r}^{\infty} \hat{u}_{n} \frac{\Gamma(n+\mu)}{\Gamma(n)} \frac{(n-1+r) !}{2 r(n-1) !} P_{n-1-r}^{r, r}(x) .
$$

Therefore,

$$
\begin{array}{r}
\left\|(1-x)^{r / 2}(1+x)^{r / 2}{ }_{-1} \mathcal{D}_{x}^{r+\mu} u(x)\right\|^{2}= \\
\int_{-1}^{1}(1-x)^{r}(1+x)^{r}\left(\sum_{n=r}^{\infty} \hat{u}_{n} \frac{\Gamma(n+\mu)}{\Gamma(n)} \frac{(n-1+r) !}{2 r(n-1) !} P_{n-1-r}^{r, r}(x)\right)^{2}= \\
\sum_{n=r}^{\infty}\left(\hat{u}_{n} \frac{\Gamma(n+\mu)}{\Gamma(n)} \frac{(n-1+r) !}{2 r(n-1) !}\right)^{2} \int_{-1}^{1}(1-x)^{r}(1+x)^{r} P_{n-1-r}^{r, r}(x) P_{n-1-r}^{r, r}(x) d x= \\
\sum_{n=r}^{\infty}\left[\hat{u}_{n} \frac{\Gamma(n+\mu)}{\Gamma(n)} \frac{(n-1+r) !}{2 r(n-1) !}\right]^{2} \frac{2^{2 r+1}((n-1) !)^{2}}{(n-1-r) !(n-1+r) !}= \\
\sum_{n=r}^{\infty}\left[\hat{u}_{n} \frac{\Gamma(n+\mu)}{\Gamma(n)}\right]^{2} \frac{2}{2 n+1} \frac{(n-1+r) !}{(n-1-r) !} .
\end{array}
$$


We also note that $\frac{(n-1+r) !}{(n-1-r) !}$ is minimized when $n=N+1$. Hence,

$$
\begin{aligned}
\left\|u-u_{N}\right\|_{U}^{2} & =\sum_{n=N+1}^{\infty}\left[\hat{u}_{n} \frac{\Gamma(n+\mu)}{\Gamma(n)}\right]^{2} \\
& \leq \sum_{n=N+1}^{\infty}\left[\hat{u}_{n} \frac{\Gamma(n+\mu)}{\Gamma(n)}\right]^{2} \frac{(n-1+r) !}{(n-1-r) !} \frac{(N-r) !}{(N+r) !} \\
& =\frac{(N-r) !}{(N+r) !} \sum_{n=N+1}^{\infty}\left[\hat{u}_{n} \frac{\Gamma(n+\mu)}{\Gamma(n)}\right]^{2} \frac{(n-1+r) !}{(n-1-r) !} \\
& =\frac{(N-r) !}{(N+r) !}\left\|(1-x)^{r / 2}(1+x)^{r / 2}{ }_{-1} \mathcal{D}_{x}^{r+\mu} u(x)\right\|^{2} \\
& \leq \frac{(N-r) !}{(N+r) !}\left\|_{-1} \mathcal{D}_{x}^{r+\mu} u(x)\right\|^{2} \\
& \leq c N^{-2 r}\left\|{ }_{-1} \mathcal{D}_{x}^{r+\mu} u(x)\right\|^{2},
\end{aligned}
$$

where $r \geq 1$ and $2 \mu \in(0,1)$. Similar steps are done for the case $2 \mu \in(1,2)$ to obtain (37) noting that in either case, $\mu$ remains between 0 and 1 .

Next, we consider the following two-dimensional problem in $\Omega=[-1,1] \times$ $[-1,1]$ :

$$
\begin{aligned}
{ }_{-1} \mathcal{D}_{x}^{2 \mu_{x}} u(x, y) & +{ }_{-1} \mathcal{D}_{y}^{2 \mu_{y}} u(x, y)=f(x, y), \quad \forall(x, y) \in \Omega, \\
u(-1, y) & =u(x,-1)=0, \quad \text { if } 0<\mu_{x}, \mu_{y}<1 / 2,
\end{aligned}
$$

whose corresponding weak form is given by

$$
\left({ }_{-1} \mathcal{D}_{x}^{\mu_{x}} u,{ }_{x} \mathcal{D}_{1}^{\mu_{x}} v\right)_{\Omega}+\left({ }_{-1} \mathcal{D}_{y}^{\mu_{y}} u,{ }_{y} \mathcal{D}_{1}^{\mu_{y}} v\right)_{\Omega}=(f, v)_{\Omega}
$$

We represent the exact solution $u$ when $2 \mu_{x}, 2 \mu_{y} \in(0,1)$, in terms of the following infinite series of tensor product Jacobi poly-fractonomials as

$$
u(x, y)=\sum_{n=1}^{\infty} \sum_{m=1}^{\infty} \hat{u}_{n m}(1+x)^{\mu_{x}} P_{n-1}^{-\mu_{x}, \mu_{x}}(x)(1+y)^{\mu_{y}} P_{m-1}^{-\mu_{y}, \mu_{y}}(y) .
$$


Hence,

$$
\begin{aligned}
{ }_{-1} \mathcal{D}_{x}^{r+\mu_{x}} u=\sum_{n=1}^{\infty} \sum_{m=1}^{\infty} \hat{u}_{n m} \frac{\Gamma\left(n+\mu_{x}\right)}{\Gamma(n)} \frac{(n-1+r) !}{2 r(n-1) !} P_{n-1-r}^{r, r}(x)(1+y)^{\mu_{y}} P_{m-1}^{-\mu_{y}, \mu_{y}}(y) \\
{ }_{-1} \mathcal{D}_{y}^{r+\mu_{y}} u=\sum_{n=1}^{\infty} \sum_{m=1}^{\infty} \hat{u}_{n m}(1+x)^{\mu_{x}} P_{n-1}^{-\mu_{x}, \mu_{x}}(x) \frac{\Gamma\left(m+\mu_{y}\right)}{\Gamma(m)} \frac{(m-1+r) !}{2 r(m-1) !} P_{m-1-r}^{r, r}(y)
\end{aligned}
$$

Moreover, taking $w_{1}(x)=(1-x)^{r / 2}(1+x)^{r / 2}$ and $w_{2}(y)=(1-y)^{-\mu_{y} / 2}(1+$ $y)^{-\mu_{y} / 2}$, we have

$$
\begin{aligned}
& \left\|w_{1}(x) w_{2}(y)_{-1} \mathcal{D}_{x}^{r+\mu_{x}} u\right\|_{L^{2}(\Omega)}^{2}= \\
& \quad \sum_{n=1}^{\infty} \sum_{m=1}^{\infty}\left[\hat{u}_{n m} \frac{\Gamma\left(n+\mu_{x}\right)}{\Gamma(n)} \frac{(n-1+r) !}{2 r(n-1) !}\right]^{2} \cdot \int_{-1}^{+1}(1-x)^{r}(1+x)^{r}\left[P_{n-1-r}^{r, r}(x)\right]^{2} d x \\
& =\sum_{n=1}^{+1}(1-y)^{-\mu_{y}}(1+y)^{\mu_{y}}\left[P_{m-1}^{-\mu_{y}, \mu_{y}}(y)\right]^{2} d y \\
& \sum_{m=1}^{\infty}\left[\hat{u}_{n m} \frac{\Gamma\left(n+\mu_{x}\right)}{\Gamma(n)}\right]^{2} \frac{2}{2 n+1} \cdot \frac{(n-1+r) !}{(n-1-r) !} \frac{2}{2 m-1} \frac{\Gamma\left(m-\mu_{y}\right) \Gamma\left(m+\mu_{y}\right)}{(m-1) ! \Gamma(m)} .
\end{aligned}
$$

Similarly,

$$
\begin{aligned}
& \left\|w_{1}(y) w_{2}(x){ }_{-1} \mathcal{D}_{y}^{r+\mu_{y}} u\right\|_{L^{2}(\Omega)}^{2}= \\
& \sum_{n=1}^{\infty} \sum_{m=1}^{\infty}\left[\hat{u}_{n m} \frac{\Gamma\left(m+\mu_{x}\right)}{\Gamma(m)}\right]^{2} \frac{2}{2 n-1} \frac{\Gamma\left(n-\mu_{x}\right) \Gamma\left(n+\mu_{x}\right)}{(n-1) ! \Gamma(n)} \frac{2}{2 m+1} \frac{(m-1+r) !}{(m-1-r) !} .
\end{aligned}
$$

We note that $u(x, y)$ can be decomposed into four contributions as

$$
u=\left(\sum_{n=1}^{N} \sum_{m=1}^{N}+\sum_{n=1}^{N} \sum_{m=N+1}^{\infty}+\sum_{n=N+1}^{\infty} \sum_{m=1}^{N}+\sum_{n=N+1}^{\infty} \sum_{m=N+1}^{\infty}\right) \hat{u}_{n m} \mathcal{P}_{n}^{\mu_{x}}(x) \mathcal{P}_{m}^{\mu_{y}}(y)
$$

or equivalently,

$$
u-u_{N}=\left(\sum_{n=1}^{N} \sum_{m=N+1}^{\infty}+\sum_{n=N+1}^{\infty} \sum_{m=1}^{N}+\sum_{n=N+1}^{\infty} \sum_{m=N+1}^{\infty}\right) \hat{u}_{n m} \mathcal{P}_{n}^{\mu_{x}}(x) \mathcal{P}_{m}^{\mu_{y}}(y)
$$


Next, we aim to bound $\left\|u-u_{N}\right\|_{U}$ in terms of higher-order derivative as

$$
\begin{aligned}
\left\|u-u_{N}\right\|_{U}^{2} & \leq\left\|\sum_{n=1}^{N} \sum_{m=N+1}^{\infty} \hat{u}_{n m} \mathcal{P}_{n}^{\mu_{x}}(x) \mathcal{P}_{m}^{\mu_{y}}(y)\right\|_{U}^{2} \\
& +\left\|\sum_{n=N+1}^{\infty} \sum_{m=1}^{N} \hat{u}_{n m} \mathcal{P}_{n}^{\mu_{x}}(x) \mathcal{P}_{m}^{\mu_{y}}(y)\right\|_{U}^{2} \\
& +\left\|\sum_{n=N+1}^{\infty} \sum_{m=N+1}^{\infty} \hat{u}_{n m} \mathcal{P}_{n}^{\mu_{x}}(x) \mathcal{P}_{m}^{\mu_{y}}(y)\right\|_{U}^{2}
\end{aligned}
$$

in which we note the symmetry between the first two terms on the right-hand side. Let us consider the second term first:

$$
\begin{array}{r}
\left\|\sum_{n=N+1}^{\infty} \sum_{m=1}^{N} \hat{u}_{n m} \mathcal{P}_{n}^{\mu_{x}}(x) \mathcal{P}_{m}^{\mu_{y}}(y)\right\|_{U}^{2}= \\
\left\|_{-1} \mathcal{D}_{x}^{\mu_{x}}\left(\sum_{n=N+1}^{\infty} \sum_{m=1}^{N} \hat{u}_{n m} \mathcal{P}_{n}^{\mu_{x}}(x) \mathcal{P}_{m}^{\mu_{y}}(y)\right)\right\|_{L^{2}(\Omega)}^{2}+ \\
\left\|_{-1} \mathcal{D}_{y}^{\mu_{y}}\left(\sum_{n=N+1}^{\infty} \sum_{m=1}^{N} \hat{u}_{n m} \mathcal{P}_{n}^{\mu_{x}}(x) \mathcal{P}_{m}^{\mu_{y}}(y)\right)\right\|_{L^{2}(\Omega)}^{2},
\end{array}
$$

where

$$
\begin{aligned}
& \left\|_{-1} \mathcal{D}_{x}^{\mu_{x}}\left(\sum_{n=N+1}^{\infty} \sum_{m=1}^{N} \hat{u}_{n m} \mathcal{P}_{n}^{\mu_{x}}(x) \mathcal{P}_{m}^{\mu_{y}}(y)\right)\right\|_{L^{2}(\Omega)}^{2}= \\
& \leq \int_{-1}^{1} \int_{-1}^{1} w_{2}(y)\left(\sum_{n=N+1}^{\infty} \sum_{m=1}^{N} \hat{u}_{n m} \frac{\Gamma\left(n+\mu_{x}\right)}{\Gamma(n)} P_{n-1}(x) \mathcal{P}_{m}^{\mu_{y}}(y)\right)^{2} \\
& \leq \sum_{n=N+1}^{\infty} \sum_{m=1}^{N}\left[\hat{u}_{n m} \frac{\Gamma\left(n+\mu_{x}\right)}{\Gamma(n)}\right]^{2} \frac{2}{2 n+1} \frac{2}{2 m-1} \frac{\Gamma\left(m-\mu_{y}\right) \Gamma\left(m+\mu_{y}\right)}{(m-1) ! \Gamma(m)} \\
& \leq \frac{(N-r) !}{(N+r) !} \sum_{n=N+1}^{\infty} \sum_{m=1}^{N}\left[\hat{u}_{n m} \frac{\Gamma\left(n+\mu_{x}\right)}{\Gamma(n)}\right]^{2} \frac{2}{2 n+1} \frac{(n-1+r) !}{(n-1-r) !} \frac{2}{2 m-1} \frac{\Gamma\left(m-\mu_{y}\right) \Gamma\left(m+\mu_{y}\right)}{(m-1) ! \Gamma(m)} \\
& \leq \frac{(N-r) !}{(N+r) !}\left\|_{-1} \mathcal{D}_{x}^{1+\mu_{x}} u\right\|_{L^{2}(\Omega)}^{2} \\
& \leq c N^{-2 r}\left\|{ }_{-1} \mathcal{D}_{x}^{1+\mu_{x}} u\right\|_{L^{2}(\Omega)}^{2} .
\end{aligned}
$$


Following similar steps, we obtain

$$
\left\|_{-1} \mathcal{D}_{y}^{\mu_{y}}\left(\sum_{n=N+1}^{\infty} \sum_{m=1}^{N} \hat{u}_{n m} \mathcal{P}_{n}^{\mu_{x}}(x) \mathcal{P}_{m}^{\mu_{y}}(y)\right)\right\|_{L^{2}(\Omega)}^{2} \leq c N^{-2 r}\left\|_{-1} \mathcal{D}_{y}^{r+\mu_{y}} u\right\|_{L^{2}(\Omega)}^{2} .
$$

Therefore, we obtain the following estimate for the second term on the righthand side of (43)

$\left\|\sum_{n=N+1}^{\infty} \sum_{m=1}^{N} \hat{u}_{n m} \mathcal{P}_{n}^{\mu_{x}}(x) \mathcal{P}_{m}^{\mu_{y}}(y)\right\|_{U}^{2} \leq \tilde{c} N^{-2 r}\left(\left\|{ }_{-1} \mathcal{D}_{x}^{r+\mu_{x}} u\right\|_{L^{2}(\Omega)}^{2}+\left\|_{-1} \mathcal{D}_{y}^{r+\mu_{y}} u\right\|_{L^{2}(\Omega)}^{2}\right)$

Moreover, by symmetry, we have the following results for the first term on the right-hand side of (43):

$\left\|\sum_{n=1}^{N} \sum_{m=N+1}^{\infty} \hat{u}_{n m} \mathcal{P}_{n}^{\mu_{x}}(x) \mathcal{P}_{m}^{\mu_{y}}(y)\right\|_{U}^{2} \leq \tilde{C} N^{-2 r}\left(\left\|{ }_{-1} \mathcal{D}_{x}^{r+\mu_{x}} u\right\|_{L^{2}(\Omega)}^{2}+\left\|_{-1} \mathcal{D}_{y}^{r+\mu_{y}} u\right\|_{L^{2}(\Omega)}^{2}\right)$

It is easy to check that $\left\|\sum_{n=N+1}^{\infty} \sum_{m=N+1}^{\infty} \hat{u}_{n m} \mathcal{P}_{n}^{\mu_{x}}(x) \mathcal{P}_{m}^{\mu_{y}}(y)\right\|_{U}^{2}$ can be bounded by the first two terms on the right-hand side, hence, by substituting (45) and (46) into (43), we finally obtain the following estimate

$$
\left\|u-u_{N}\right\|_{U}^{2} \leq C N^{-2 r}\left(\left\|_{-1} \mathcal{D}_{x}^{r+\mu_{x}} u\right\|_{L^{2}(\Omega)}^{2}+\left\|_{-1} \mathcal{D}_{y}^{r+\mu_{y}} u\right\|_{L^{2}(\Omega)}^{2}\right) .
$$

Such an error estimate can be isotropically tensor producted up for higherdimensional problems to get the following estimate

$$
\left\|u-u_{N}\right\|_{U}^{2} \leq C N^{-2 r}\left(\left\|_{0} \mathcal{D}_{t}^{r+\tau} u\right\|_{L^{2}(\Omega)}^{2}+\sum_{j=1}^{d}\left\|{ }_{-1} \mathcal{D}_{x_{j}}^{r+\mu_{j}} u\right\|_{L^{2}(\Omega)}^{2}\right),
$$

in which $N$ denotes the number of terms in the expansion in all $(d+1)$ dimensions.

Remark 3.5. Since the inf-sup condition holds (see Theorem 3.2), by the Banach-Nečas-Babuška theorem [48], the error in the numerical scheme is less than or equal to a constant times the projection error. Choosing the projection $u_{N}$ in Theorem 3.4, we conclude the spectral accuracy of the scheme. 


\subsection{Implementation of PG Spectral Method}

We now seek the solution to (8) in terms of a linear combination of elements in the space $U_{N}$ of the form

$$
u_{N}(x, t)=\sum_{n=\lceil 2 \tau\rceil}^{\mathcal{N}} \sum_{m_{1}=\left\lceil 2 \mu_{1}\right\rceil}^{\mathcal{M}_{1}} \ldots \sum_{m_{d}=\left\lceil 2 \mu_{d}\right\rceil}^{\mathcal{M}_{d}} \hat{u}_{n, m_{1}, \cdots, m_{d}}\left[\psi_{n}^{\tau}(t) \prod_{j=1}^{d} \phi_{m_{j}}^{\mu_{j}}\left(x_{j}\right)\right]
$$

in $\Omega$. Next, we require the corresponding residual

$$
R_{N}\left(t, x_{1}, \cdots, x_{d}\right)={ }_{0} \mathcal{D}_{t}^{2 \tau}\left(u_{N}\right)+\sum_{j=1}^{d} c_{j} a_{a_{j}} \mathcal{D}_{x_{j}}^{2 \mu_{j}}\left(u_{N}\right)+\gamma u_{N}-f
$$

to be $L^{2}$-orthogonal to the elements in $v_{N} \in V_{N}$, which leads to the finitedimensional variational form given in (17). Specifically, by choosing $v_{N}=$ $\Psi_{r}^{\mu_{t}}(t) \prod_{j=1}^{d} \Phi_{k_{j}}^{\mu_{x_{j}}}\left(x_{j}\right)$, when $r=\lceil 2 \tau\rceil, \ldots, \mathcal{N}$ and $k_{j}=\left\lceil 2 \mu_{j}\right\rceil, \ldots, \mathcal{M}_{j}$, we obtain

$$
\begin{array}{r}
\sum_{n=\lceil 2 \tau\rceil}^{\mathcal{N}} \sum_{m_{1}=\left\lceil 2 \mu_{1}\right\rceil}^{\mathcal{M}_{1}} \cdots \sum_{m_{d}=\left\lceil 2 \mu_{d}\right\rceil}^{\mathcal{M}_{d}} \hat{u}_{n, m_{1}, \cdots, m_{d}}\left(\left\{S_{\tau}\right\}_{r, n}\left\{M_{\mu_{1}}\right\}_{k_{1}, m_{1}} \cdots\left\{M_{\mu_{d}}\right\}_{k_{d}, m_{d}}(5)\right. \\
+\sum_{j=1}^{d} c_{j}\left[\left\{M_{\tau}\right\}_{r, n}\left\{M_{\mu_{1}}\right\}_{k_{1}, m_{1}} \cdots\left\{S_{\mu_{j}}\right\}_{k_{j}, m_{j}} \cdots\left\{M_{\mu_{d}}\right\}_{k_{d}, m_{d}}\right] \\
\left.+\gamma\left\{M_{\tau}\right\}_{r, n}\left\{M_{\mu_{1}}\right\}_{k_{1}, m_{1}} \cdots\left\{M_{\mu_{d}}\right\}_{k_{d}, m_{d}}\right)=F_{r, k_{1}, \cdots, k_{d}},
\end{array}
$$

where $S_{\tau}$ and $M_{\tau}$ denote, respectively, the temporal stiffness and mass matrices, whose entries are defined as

$$
\left\{S_{\tau}\right\}_{r, n}=\int_{0}^{T}{ }_{0} \mathcal{D}_{t}^{\tau}\left(\psi_{n}^{\tau} \circ \eta\right)(t){ }_{t} \mathcal{D}_{T}^{\tau}\left(\Psi_{r}^{\tau} \circ \eta\right)(t) d t,
$$

and

$$
\left\{M_{\tau}\right\}_{r, n}=\int_{0}^{T}\left(\Psi_{r}^{\tau} \circ \eta\right)(t)\left(\psi_{n}^{\tau} \circ \eta\right)(t) d t .
$$

Moreover, $S_{\mu_{j}}$ and $M_{\mu_{j}}, j=1,2, \cdots, d$, are the corresponding spatial stiffness and mass matrices

$$
\left\{S_{\mu_{j}}\right\}_{k_{j}, m_{j}}=\int_{a_{j}}^{b_{j}}{ }_{a_{j}} \mathcal{D}_{x_{j}}^{\mu_{j}}\left(\phi_{m_{j}}^{\mu_{j}} \circ \xi_{j}\right)\left(x_{j}\right)_{x_{j}} \mathcal{D}_{b_{j}}^{\mu_{j}}\left(\Phi_{k_{j}}^{\mu_{j}} \circ \xi_{j}\right)\left(x_{j}\right) d x_{j}
$$


and

$$
\left\{M_{\mu_{j}}\right\}_{k_{j}, m_{j}}=\int_{a_{j}}^{b_{j}}\left(\Phi_{k_{j}}^{\mu_{j}} \circ \xi_{j}\right)\left(x_{j}\right)\left(\phi_{m_{j}}^{\mu_{j}} \circ \xi_{j}\right)\left(x_{j}\right) d x_{j},
$$

respectively, to be exactly computed in the Appendix. Moreover, $F_{r, k_{1}, \cdots, k_{d}}$ is

$$
\int_{\Omega} f\left(t, x_{1}, \cdots, x_{d}\right)\left(\Psi_{r}^{\tau} \circ \eta\right)(t) \prod_{j=1}^{d}\left(\Phi_{k_{j}}^{\mu_{j}} \circ \xi_{j}\right)\left(x_{j}\right) d \Omega .
$$

Assuming that all the aforementioned stiffness and mass matrices are symmetric, we can render the linear system (51) as the following general Lyapunov equation

$$
\begin{gathered}
\left(S_{\tau} \otimes M_{\mu_{1}} \otimes M_{\mu_{2}} \cdots \otimes M_{\mu_{d}}\right. \\
+\sum_{j=1}^{d} c_{j}\left[M_{\tau} \otimes M_{\mu_{1}} \otimes \cdots \otimes M_{\mu_{j-1}} \otimes S_{\mu_{j}} \otimes M_{\mu_{j+1}} \cdots \otimes M_{\mu_{d}}\right] \\
\left.\quad+\gamma M_{\tau} \otimes M_{\mu_{1}} \otimes M_{\mu_{2}} \cdots \otimes M_{\mu_{d}}\right) \mathcal{U}=F
\end{gathered}
$$

in which $\otimes$ represents the Kronecker product, $F$ denotes the multi-dimensional load matrix whose entries given in (52), and $\mathcal{U}$ is the corresponding multidimensional matrix of unknown coefficients whose entries are $\hat{u}_{n, m_{1}, \cdots, m_{d}}$.

In the Appendix, we investigate the properties of the aforementioned matrices in addition to presenting efficient ways of deriving the stiffness matrices explicitly and computing the mass matrices exactly through proper quadrature rules.

\subsection{A New Fast FPDE Solver}

So far, we have formulated a suitable Petrov-Galerkin variational framework for the general $(1+d)$-dimensional FPDE, given in $(8)$, by choosing proper basis and test functions. The main advantage of such framework is that we can explicitly obtain the corresponding stiffness matrices to be symmetric diagonal/tridiagonal, and moreover, to exactly compute the mass matrices, which we showed to be symmetric. The following result better highlights the benefit of this scheme, where we formulate a closed-form solution for the Lyapunov system (53) in terms of the generalised eigensolutions that can be computed very efficiently. 
Theorem 3.6. Let $\left\{\vec{e}_{m_{j}}^{\mu_{j}}, \lambda_{m_{j}}^{\mu_{j}}\right\}_{m_{j}=\left\lceil 2 \mu_{j}\right\rceil}^{\mathcal{M}_{j}}$ be the set of general eigen-solutions of the spatial mass matrix $M_{\mu_{j}}$ with respect to the stiffness matrix $S_{\mu_{j}}$. Moreover, let us assume that $\left\{\vec{e}_{n}^{\tau}, \lambda_{n}^{\tau}\right\}_{n=\lceil 2 \tau\rceil}^{\mathcal{N}}$ are the set of general eigen-solutions of the temporal mass matrix $M_{\tau}$ with respect to the stiffness matrix $S_{\tau}$. (I) if $d>1$, then the multi-dimensional matrix of unknown solution $\mathcal{U}$ is explicitly obtained as

$$
\mathcal{U}=\sum_{n=\lceil 2 \tau\rceil}^{\mathcal{N}} \sum_{m_{1}=\left\lceil 2 \mu_{1}\right\rceil}^{\mathcal{M}_{1}} \cdots \sum_{m_{d}=\left\lceil 2 \mu_{d}\right\rceil}^{\mathcal{M}_{d}} \kappa_{n, m_{1}, \cdots, m_{d}} \vec{e}_{n}^{\tau} \otimes \vec{e}_{m_{1}}^{\mu_{1}} \otimes \cdots \otimes \vec{e}_{m_{d}}^{\mu_{d}},
$$

where the unknown $\kappa_{n, m_{1}, \cdots, m_{d}}$ are given by

$$
\kappa_{n, m_{1}, \cdots, m_{d}}=\frac{\left(\vec{e}_{n}^{\tau} \vec{e}_{m_{1}}^{\mu_{1}} \cdots \vec{e}_{m_{d}}^{\mu_{d}}\right) F}{\left[\left(\vec{e}_{n}^{\tau^{T}} S_{\tau} \vec{e}_{n}^{\tau}\right) \prod_{j=1}^{d}\left(\vec{e}_{m_{j}}^{\mu_{j}^{T}} S_{\mu_{j}} \vec{e}_{m_{j}}^{\mu_{j}}\right)\right] \Lambda_{n, m_{1}, \cdots, m_{d}}} .
$$

in which the numerator represents the standard multi-dimensional inner product, and $\Lambda_{n, m_{1}, \cdots, m_{d}}$ are obtained in terms of the eigenvalues of all mass matrices as

$$
\Lambda_{n, m_{1}, \cdots, m_{d}}=\left[\left(1+\gamma \lambda_{n}^{\tau}\right) \prod_{j=1}^{d} \lambda_{m_{j}}^{\mu_{j}}+\lambda_{n}^{\tau} \sum_{j=1}^{d} c_{j}\left(\prod_{s=1, s \neq j}^{d} \lambda_{m_{s}}^{\mu_{s}}\right)\right] .
$$

(II) If $d=1$, then the two-dimensional matrix of the unknown solution $\mathcal{U}$ is obtained as

$$
\mathcal{U}=\sum_{n=\lceil 2 \tau\rceil}^{\mathcal{N}} \sum_{m_{1}=\left\lceil 2 \mu_{1}\right\rceil}^{\mathcal{M}_{1}} \kappa_{n, m_{1}} \vec{e}_{n}^{\tau} \vec{e}_{m_{1}}^{\mu_{1}^{T}}
$$

where $\kappa_{n, m_{1}}$ is explicitly obtained as

$$
\kappa_{n, m_{1}}=\frac{\vec{e}_{n}^{\tau^{T}} F \vec{e}_{m_{1}}^{\mu_{1}}}{\left(\vec{e}_{n}^{\tau^{T}} S_{\tau} \vec{e}_{n}^{\tau}\right)\left(\vec{e}_{m_{1}}^{\mu_{1}^{T}} S_{\mu_{1}} \vec{e}_{m_{1}}^{\mu_{1}}\right)\left[\lambda_{m_{1}-1}^{\mu_{1}}+c_{1} \lambda_{n}^{\tau}+\gamma \lambda_{n}^{\tau} \lambda_{m_{1}}^{\mu_{1}}\right]} .
$$

Proof. Let us consider the following generalised eigenvalue problems

$$
\begin{aligned}
M_{\mu_{j}} \vec{e}_{m_{j}}^{\mu_{j}} & =\lambda_{m_{j}}^{\mu_{j}} S_{\mu_{j}} \vec{e}_{m_{j}}^{\mu_{j}}, \quad m_{j}=\left\lceil 2 \mu_{j}\right\rceil, \cdots, \mathcal{M}_{j}, \quad j=1,2, \cdots, d, \\
M_{\tau} \vec{e}_{n}^{\tau} & =\lambda_{n}^{\tau} S_{\tau} \vec{e}_{n}^{\tau}, \quad n=\lceil 2 \tau\rceil, 2, \cdots, \mathcal{N} .
\end{aligned}
$$


We aim to represent the unknown coefficient matrix $\mathcal{U}$ in the expansion (49) in terms of the spatial and temporal eigenvectors as

$$
\mathcal{U}=\sum_{n=\lceil 2 \tau\rceil}^{\mathcal{N}} \sum_{m_{1}=\left\lceil 2 \mu_{1}\right\rceil}^{\mathcal{M}_{1}} \cdots \sum_{m_{d}=\left\lceil 2 \mu_{d}\right\rceil}^{\mathcal{M}_{d}} \kappa_{n, m_{1}, \cdots, m_{d}} \vec{e}_{n}^{\tau} \otimes \vec{e}_{m_{1}}^{\mu_{1}} \otimes \cdots \otimes \vec{e}_{m_{d}}^{\mu_{d}},
$$

where the unknown $\kappa_{n, m_{1}, \cdots, m_{d}}$ are obtained as follows. We first take the multi-dimensional inner product of $\vec{e}_{q}^{\tau} \vec{e}_{p_{1}}^{\mu_{1}} \cdots \vec{e}_{p_{d}}^{\mu_{d}}$ on both sides of the Lyapunov equation (53) to obtain

$$
\begin{aligned}
&\left(\vec{e}_{q}^{\tau} \vec{e}_{p_{1}}^{\mu_{1}} \vec{e}_{p_{2}}^{\mu_{2}} \cdots \vec{e}_{p_{d}}^{\mu_{d}}\right)\left[S_{\tau} \otimes M_{\mu_{1}} \otimes \cdots \otimes M_{\mu_{d}}\right. \\
&+\sum_{j=1}^{d} c_{j}\left[M_{\tau} \otimes M_{\mu_{1}} \otimes \cdots \otimes M_{\mu_{j-1}} \otimes S_{\mu_{j}} \otimes M_{\mu_{j+1}} \cdots \otimes M_{\mu_{d}}\right] \\
&\left.\quad+\gamma M_{\tau} \otimes M_{\mu_{1}} \otimes \cdots \otimes M_{\mu_{d}}\right] \mathcal{U}=\left(\vec{e}_{q}^{\tau} \vec{e}_{p_{1}}^{\mu_{1}} \cdots \vec{e}_{p_{d}}^{\mu_{d}}\right) F
\end{aligned}
$$

Next, we substitute (58) and re-arrange the terms to obtain

$$
\begin{aligned}
& \sum_{n=\lceil 2 \tau\rceil\rceil}^{\mathcal{N}} \sum_{m_{1}=\left\lceil 2 \mu_{1}\right\rceil}^{\mathcal{M}_{1}} \cdots \sum_{m_{d}=\left\lceil 2 \mu_{d}\right\rceil}^{\mathcal{M}_{d}} \kappa_{n, m_{1}, \cdots, m_{d}} \\
& \left(\begin{array}{cccc}
\vec{e}_{q}^{\tau^{T}} S_{\tau} \vec{e}_{n}^{\tau} & \vec{e}_{p_{1}}^{\mu_{j}^{T}} M_{\mu_{1}} \vec{e}_{m_{1}}^{\mu_{j}} \quad \cdots \quad \vec{e}_{p_{d}}^{\mu_{j}^{T}} M_{\mu_{d}} \vec{e}_{m_{d}}^{\mu_{j}}
\end{array}\right. \\
& +\sum_{j=1}^{d} c_{j} \vec{e}_{q}^{\tau^{T}} M_{\tau} \vec{e}_{n}^{\tau} \quad \vec{e}_{p_{1}}^{\mu_{j}^{T}} M_{\mu_{1}} \vec{e}_{m_{1}}^{\mu_{j}} \quad \cdots \quad \vec{e}_{p_{j}}^{\mu_{j}^{T}} S_{\mu_{j}} \vec{e}_{m_{j}}^{\mu_{j}} \quad \vec{e}_{p_{j+1}}^{\mu_{j+1}^{T}} M_{\mu_{j+1}} \vec{e}_{m_{j+1}}^{\mu_{j+1}} \quad \vec{e}_{p_{d}}^{\mu_{j}^{T}} M_{\mu_{d}} \vec{e}_{m_{d}}^{\mu_{j}} \\
& \left.+\gamma \vec{e}_{q}^{\tau^{T}} M_{\tau} \vec{e}_{n}^{\tau} \quad \vec{e}_{p_{1}}^{\mu_{j}^{T}} M_{\mu_{1}} \vec{e}_{m_{1}}^{\mu_{j}} \quad \vec{e}_{p_{2}}^{\mu_{j}^{T}} M_{\mu_{2}} \vec{e}_{m_{2}}^{\mu_{j}} \quad \ldots \quad \vec{e}_{p_{d}}^{\mu_{j}^{T}} M_{\mu_{d}} \vec{e}_{m_{d}}^{\mu_{j}}\right) \\
& =\left(\vec{e}_{q}^{\tau} \vec{e}_{p_{1}}^{\mu_{1}} \vec{e}_{p_{2}}^{\mu_{2}} \cdots \vec{e}_{p_{d}}^{\mu_{d}}\right) F
\end{aligned}
$$


where we recall that $M_{\mu_{j}} \vec{e}_{m_{j}}^{\mu_{j}}=\left(\lambda_{m_{j}}^{\mu_{j}} S_{\mu_{j}} \vec{e}_{m_{j}}^{\mu_{j}}\right)$ and $M_{\tau} \vec{e}_{n}^{\tau}=\left(\lambda_{n}^{\tau} S_{\tau} \vec{e}_{n}^{\tau}\right)$. Hence,

$$
\begin{aligned}
& \sum_{n=\lceil 2 \tau\rceil}^{\mathcal{N}} \sum_{m_{1}=\left\lceil 2 \mu_{1}\right\rceil}^{\mathcal{M}_{1}} \cdots \sum_{m_{d}=\left\lceil 2 \mu_{d}\right\rceil}^{\mathcal{M}_{d}} \kappa_{n, m_{1}, \cdots, m_{d}}
\end{aligned}
$$

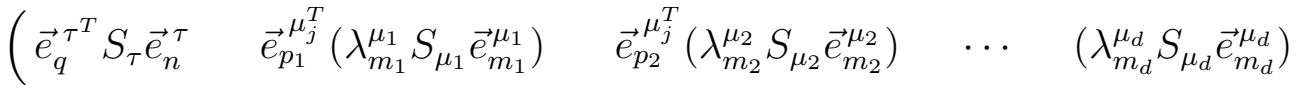

$$
\begin{aligned}
& +\sum_{j=1}^{d} c_{j} \vec{e}_{q}^{\tau^{T}}\left(\lambda_{n}^{\tau} S_{\tau} \vec{e}_{n}^{\tau}\right) \quad \vec{e}_{p_{1}}^{\mu_{j}^{T}}\left(\lambda_{m_{1}}^{\mu_{1}} S_{\mu_{1}} \vec{e}_{m_{1}}^{\mu_{1}}\right) \\
& \cdots \quad \vec{e}_{p_{j}}^{\mu_{j}^{T}} S_{\mu_{j}} \vec{e}_{m_{j}}^{\mu_{j}} \quad \vec{e}_{p_{j+1}}^{\mu_{j+1}^{T}}\left(\lambda_{m_{j+1}}^{\mu_{j}+1} S_{\mu_{j+1}} \vec{e}_{m_{j+1}}^{\mu_{j+1}}\right) \cdots \vec{e}_{p_{d}}^{\mu_{j}^{T}}\left(\lambda_{m_{d}}^{\mu_{d}} S_{\mu_{d}} \vec{e}_{m_{d}}^{\mu_{d}}\right) \\
& \left.+\gamma \vec{e}_{q}^{\tau^{T}}\left(\lambda_{n}^{\tau} S_{\tau} \vec{e}_{n}^{\tau}\right) \quad \vec{e}_{p_{1}}^{\mu_{j}^{T}}\left(\lambda_{m_{1}}^{\mu_{1}} S_{\mu_{1}} \vec{e}_{m_{1}}^{\mu_{1}}\right) \quad \vec{e}_{p_{2}}^{\mu_{j}^{T}}\left(\lambda_{m_{2}}^{\mu_{2}} S_{\mu_{2}} \vec{e}_{m_{2}}^{\mu_{2}}\right) \cdots\left(\lambda_{m_{d}}^{\mu_{d}} S_{\mu_{d}} \vec{e}_{m_{d}}^{\mu_{d}}\right)\right) \\
& =\left(\vec{e}_{q}^{\tau} \vec{e}_{p_{1}}^{\mu_{1}} \vec{e}_{p_{2}}^{\mu_{2}} \cdots \vec{e}_{p_{d}}^{\mu_{d}}\right) F .
\end{aligned}
$$

or alternatively,

$$
\begin{aligned}
& \sum_{n=\lceil 2 \tau\rceil}^{\mathcal{N}} \sum_{m_{1}=\left\lceil 2 \mu_{1}\right\rceil}^{\mathcal{M}_{1}} \cdots \sum_{m_{d}=\left\lceil 2 \mu_{d}\right\rceil}^{\mathcal{M}_{d}} \kappa_{n, m_{1}, \cdots, m_{d}}\left(\vec{e}_{q}^{\tau^{T}} S_{\tau} \vec{e}_{n}^{\tau}\right)\left(\vec{e}_{p_{1}}^{\mu_{j}^{T}} S_{\mu_{1}} \vec{e}_{m_{1}}^{\mu_{1}}\right) \cdots\left(\vec{e}_{p_{d}}^{\mu_{d}^{T}} S_{\mu_{d}} \vec{e}_{m_{d}}^{\mu_{d}}\right) \\
& \quad \times\left[\left(1+\gamma \lambda_{n}^{\tau}\right) \prod_{j=1}^{d} \lambda_{m_{j}}^{\mu_{j}}+\lambda_{n}^{\tau} \sum_{j=1}^{d} c_{j}\left(\prod_{s=1, s \neq j}^{d} \lambda_{m_{s}}^{\mu_{s}}\right)\right]=\left(\vec{e}_{q}^{\tau} \vec{e}_{p_{1}}^{\mu_{1}} \vec{e}_{p_{2}}^{\mu_{2}} \cdots \vec{e}_{p_{d}}^{\mu_{d}}\right) F
\end{aligned}
$$

and since the spatial and temporal stiffness matrices $S_{\mu_{j}}$ and $S_{\tau}$ are diagonal (see Appendix), then $\left(\vec{e}_{q}^{\tau^{T}} S_{\tau} \vec{e}_{n}^{\tau}\right)=0$ if $q \neq n$, also $\left(\vec{e}_{p_{j}}^{\mu_{j}^{T}} S_{\mu_{j}} \vec{e}_{m_{j}}^{\mu_{j}}\right)=0$, if $p_{j} \neq m_{j}$, which completes the proof for the case $d>1$. Following similar steps for the two-dimensional problem in the $t$ - $\mu_{1}$ domain, it is easy to see that if $d=1$, the relationship for $\kappa$ can be derived as

$$
\kappa_{q, p_{1}}=\frac{\vec{e}_{q}^{\tau^{T}} F \vec{e}_{p_{1}}^{\mu_{1}}}{\left(\vec{e}_{q}^{\tau^{T}} S_{\tau} \vec{e}_{q}^{\tau}\right)\left(\vec{e}_{p_{1}}^{\mu_{1} T} S_{\mu_{1}} \vec{e}_{p_{1}}^{\mu_{1}}\right)\left[\lambda_{p}^{\mu_{1}}+c_{\mu_{1}} \lambda_{q}^{\tau}+\gamma \lambda_{q}^{\tau} \lambda_{p}^{\mu_{1}}\right]} .
$$

Remark 3.7. If $\mu_{j}=\mu \neq \tau$ and $\mathcal{M}_{j}=\mathcal{M}, j=1,2, \cdots, d$, then the complexity of the calculations of (56) and (57) reduces to two linear generalised eigen-problems for space and time. Moreover, if $\mu=\tau$ and $\mathcal{M}=\mathcal{N}$, then we only need to solve a single one-dimensional eigen-problem $M_{\mu} \vec{e}_{q}=\lambda_{q} S_{\mu} \vec{e}_{q}$ once. 
Remark 3.8. For time-independent (steady-state) problems, where the timefractional derivative vanishes in (8), the same general framework holds. For such problems, the time-dependent basis and test functions in $U_{N}$ and $V_{N}$ consequently vanish, and we construct the $d$-dimensional basis space $\mathcal{U}_{N}$ in $\Omega=\left[a_{1}, b_{1}\right] \times \cdots \times\left[a_{d}, b_{d}\right]$ as

$$
\mathcal{U}_{N}=\operatorname{span}\left\{\prod_{j=1}^{d}\left(\phi_{m_{j}}^{\mu_{j}} \circ \xi_{j}\right)\left(x_{j}\right): m_{j}=\left\lceil 2 \mu_{j}\right\rceil, \ldots, \mathcal{M}_{j}\right\}
$$

where we seek the solution in terms of elements in the space $\mathcal{U}_{N}$ of the form

$$
u_{N}\left(\mu_{1}, \cdots, \mu_{d}\right)=\sum_{m_{1}=\left\lceil 2 \mu_{1}\right\rceil}^{\mathcal{M}_{1}} \cdots \sum_{m_{d}=\left\lceil 2 \mu_{d}\right\rceil}^{\mathcal{M}_{d}} \hat{u}_{m_{1}, \cdots, m_{d}} \prod_{j=1}^{d}\left(\phi_{m_{j}}^{\mu_{j}} \circ \xi_{j}\right)\left(x_{j}\right)
$$

and test the problem against the elements in

$$
\mathcal{V}_{N}=\operatorname{span}\left\{\prod_{j=1}^{d}\left(\Phi_{k_{j}}^{\mu_{j}} \circ \xi_{j}\right)\left(x_{j}\right): k_{j}=\left\lceil 2 \mu_{j}\right\rceil, \ldots, \mathcal{M}_{j}\right\}
$$

Subsequently, we obtain a similar Lyapunov equation as in (53) where $M_{\tau}$ no longer appears, however, $M_{\mu_{j}}$ and $S_{\mu_{j}}$ possess all the properties presented in Theorems (6.1) and (6.3) in Appendix.

Lemma 3.9. If $d>1$, in the absence of the fractional time-derivative in (8), i.e., when $S_{\tau}$ vanishes, we obtain the matrix of unknown solution $\mathcal{U}$ in (61) as

$$
\mathcal{U}=\sum_{m_{1}=\left\lceil 2 \mu_{1}\right\rceil}^{\mathcal{M}_{1}} \cdots \sum_{m_{d}=\left\lceil 2 \mu_{d}\right\rceil}^{\mathcal{M}_{d}} \kappa_{m_{1}, \cdots, m_{d}} \vec{e}_{m_{1}}^{\mu_{1}} \otimes \cdots \otimes \vec{e}_{m_{d}}^{\mu_{d}},
$$

where the unknown $\kappa_{m_{1}, \cdots, m_{d}}$ is given by

$$
\kappa_{m_{1}, \cdots, m_{d}}=\frac{\left(\vec{e}_{m_{1}}^{\mu_{1}} \cdots \vec{e}_{m_{d}}^{\mu_{d}}\right) F}{\left[\prod_{j=1}^{d}\left(\vec{e}_{m_{j}}^{\mu_{j}^{T}} S_{\mu_{j}} \vec{e}_{m_{j}}^{\mu_{j}}\right)\right]\left[\gamma \prod_{j=1}^{d} \lambda_{m_{j}}^{\mu_{j}}+\sum_{j=1}^{d} c_{j}\left(\prod_{s=1, s \neq j}^{d} \lambda_{m_{s}}^{\mu_{s}}\right)\right]}
$$

Proof. It follows the proof in Theorem 3.6. 


\subsection{Computational Considerations}

In Theorem 3.6, we assume that the eigenvectors and eigenvalues of each mass matrix with respect to the corresponding stiffness matrices are known. Therefore, employing the PG spectral method in a $(1+d)$-dimensional problem when $(1+d) \geq 2$ leads to efficient computations. Otherwise, the computational cost of the eigensolver, which is $\mathcal{O}\left(N^{3}\right)$ in practice, becomes dominant. As we shall demonstrate, this approach appears to be even more beneficial as $(1+d)$ increases. In fact, the cost of the fast FPDE solver is associated with the following two steps: (i) the computation of $\kappa_{n, m_{1}, \cdots, m_{d}}$ in (55), and (ii) the cost of representing $\mathcal{U}$ in (54). In what follows, we show that the computational complexity of mathematical operations in our PG spectral method is $\mathcal{O}\left(N^{2+d}\right)$, the dimension of the space-time domain $\Omega$, and if we assume $\mathcal{N}=\mathcal{M}_{1}=\cdots=\mathcal{M}_{d}$.

Step (i): In order to compute the $(1+d)$-dimensional array $\kappa$ in $(55)$, we need to first calculate the numerator

$$
\left(\vec{e}_{q}^{\tau} \vec{e}_{p_{1}}^{\mu_{1}} \cdots \vec{e}_{p_{d}}^{\mu_{d}}\right) F=\sum_{i=\lceil 2 \tau\rceil}^{\mathcal{N}} \sum_{s_{1}=\left\lceil 2 \mu_{1}\right\rceil}^{\mathcal{M}_{1}} \cdots \sum_{s_{d}=\left\lceil 2 \mu_{d}\right\rceil}^{\mathcal{M}_{d}}\left\{\vec{e}_{q}^{\tau}\right\}_{i}\left\{\vec{e}_{p_{1}}^{\mu_{1}}\right\}_{s_{1}} \cdots\left\{\vec{e}_{p_{d}}^{\mu_{d}}\right\}_{s_{d}}\{F\}_{i, s_{1}, \cdots, s_{d}},(64
$$

for which naive computations for all the entries leads to a computational complexity $\mathcal{O}\left(N^{2(1+d)}\right)$ that can be intractable when $d$ increases. Alternatively, by performing sum-factorization (see [20]), the operation counts can be reduced to $\mathcal{O}\left(N^{2+d}\right)$, including the time-dimension in our calculations. Following this technique we re-write the inner-product as

$$
\begin{aligned}
\left(\vec{e}_{q}^{\tau} \vec{e}_{p_{1}}^{\mu_{1}} \cdots \vec{e}_{p_{d}}^{\mu_{d}}\right) F & = \\
\sum_{i=\lceil 2 \tau\rceil}^{\mathcal{N}}\left\{\vec{e}_{q}^{\tau}\right\}_{i} \sum_{s_{1}=\left\lceil 2 \mu_{1}\right\rceil}^{\mathcal{M}_{1}}\left\{\vec{e}_{p_{1}}^{\mu_{1}}\right\}_{s_{1}} & \cdots \sum_{s_{d-1}=\left\lceil 2 \mu_{d-1}\right\rceil}^{\mathcal{M}_{d-1}}\left\{\vec{e}_{p_{d-1}}^{\mu_{d-1}}\right\}_{s_{d-1}} \sum_{s_{d}=\left\lceil 2 \mu_{d}\right\rceil}^{\mathcal{M}_{d}}\left\{\vec{e}_{p_{d}}^{\mu_{d}}\right\}_{s_{d}}\{F\}_{i, s_{1}, \cdots, s_{d}},
\end{aligned}
$$

in which we separately obtain the inner-most sum as

$$
\mathcal{F}_{i, s_{1}, \cdots, s_{d-1}, p_{d}}^{d}=\sum_{s_{d}=\left\lceil 2 \mu_{d}\right\rceil}^{\mathcal{M}_{d}}\left\{\vec{e}_{p_{d}}^{\mu_{d}}\right\}_{s_{d}}\{F\}_{i, s_{1}, \cdots, s_{d}}
$$

and similarly we write the second inner-most sum as

$$
\mathcal{F}_{i, s_{1}, \cdots, s_{d-2}, p_{d-1}, p_{d}}^{d-1}=\sum_{s_{d-1}=\left\lceil 2 \mu_{d-1}\right\rceil}^{\mathcal{M}_{d-1}}\left\{\vec{e}_{p_{d-1}}^{\mu_{d-1}}\right\}_{s_{d-1}} \mathcal{F}_{i, s_{1}, \cdots, s_{d-1}, p_{d}}^{d} .
$$


Finally, we recursively obtain

$$
\mathcal{F}_{i, p_{1}, \cdots, p_{d}}^{1}=\sum_{s_{1}=\left\lceil 2 \mu_{1}\right\rceil}^{\mathcal{M}_{1}}\left\{\vec{e}_{p_{1}}^{\mu_{1}}\right\}_{s_{1}} \mathcal{F}_{i, p_{1}, p_{2}, \cdots, p_{d}}^{2} .
$$

We note that the operation count in computing the entries of $\mathcal{F}_{i, s_{1}, \cdots, s_{j-1}, p_{j}, \cdots, p_{d}}^{j}$ in each recursion is $\mathcal{O}\left(N^{2+d}\right)$. Now, by substituting (68) back into (65), we obtain the whole inner-product as

$$
\left(\vec{e}_{q}^{\tau} \vec{e}_{p_{1}}^{\mu_{1}} \cdots \vec{e}_{p_{d}}^{\mu_{d}}\right) F=\sum_{i=\lceil 2 \tau\rceil}^{\mathcal{N}}\left\{\vec{e}_{q}^{\tau}\right\}_{i} \mathcal{F}_{i, p_{1}, \cdots, p_{d}}^{1},
$$

which is again of complexity $\mathcal{O}\left(N^{2+d}\right)$. We observe that the total computational complexity of evaluating the inner product is $\mathcal{O}\left(N^{2+d}\right)$. Moreover, the operation count for computing the denumerator in (55) and for each entry of $\kappa_{n, m_{1}, \cdots, m_{d}}$ is $\mathcal{O}(N)$. This is true since the stiffness matrix is either diagonal or tridiagonal due to the choice of our poly-fractonomial bases. Hence, the total complexity for computing the denumerator is again $\mathcal{O}\left(N^{2+d}\right)$. We recall that we have already included the time-dimension into account, i.e., the space-time domain $\Omega \subset \mathbb{R}^{1+d}$. Hence, $\kappa$ in (55) is obtained with cost $\mathcal{O}\left(N^{2+d}\right)$.

Step (ii): In the computation of (54), we observe that sum-factorization technique helps to reduce the complexity to $\mathcal{O}\left(N^{2+d}\right)$.

\section{Special FPDEs and Numerical Tests}

In section 3, we introduced general $(1+d)$-dimensional linear FPDEs, for which we developed a general Petrov-Galerkin spectral method in addition to the general fast solver. Here, we reduce this general framework to the special well-known (i) hyperbolic FPDEs such as the fractional advection equation, (ii) parabolic FPDEs such as the fractional sub-diffusion problems, and (iii) elliptic FPDEs such as the fractional Helmholtz/Poisson equations. In the following numerical examples, we carry out the spatial/temporal $p$-refinement test via fixing correspondingly the temporal/spatial expansion order fixed at 15. 

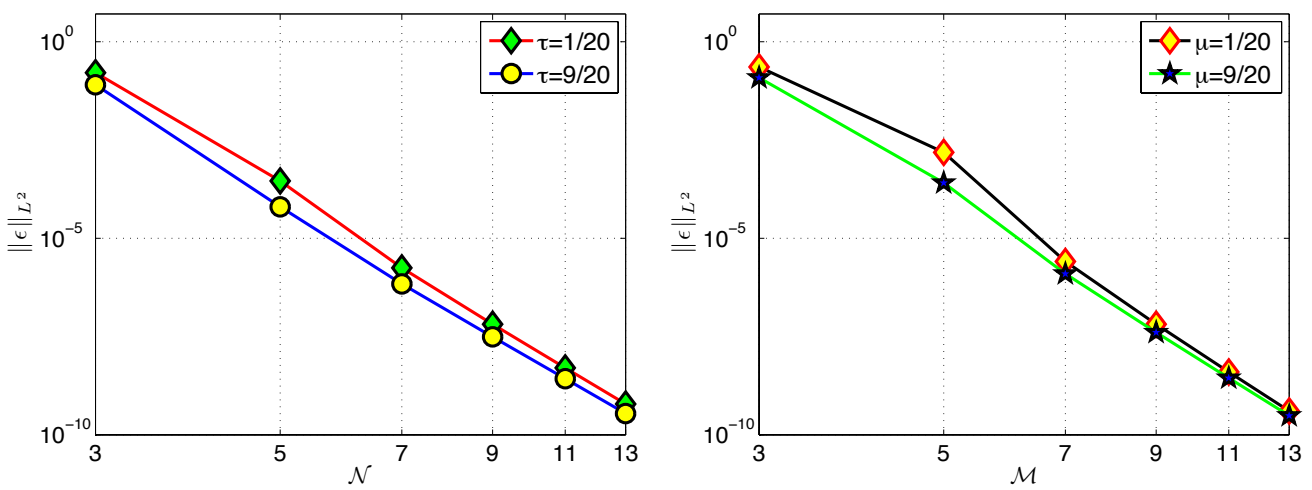

Figure 1: TSFA, temporal/spatial p-refinement: $\log$-log $L^{2}$-error versus temporal and spatial expansion orders $\mathcal{N}, \mathcal{M}$. In the temporal $p$-refinement $\tau=1 / 20$ and $9 / 20$ while $\mu=1 / 4$, also in the spatial $p$-refinement, the spatial orders $\mu=1 / 20$ and $9 / 20$ while $\tau=1 / 4$. Here, the exact solution is $u^{e x t}(x, t)=t^{6+2 / 7}(1+x)^{6+3 / 4}$.

\subsection{Hyperbolic FPDEs}

We consider the following hyperbolic FPDE

$$
{ }_{0} \mathcal{D}_{t}^{2 \tau} u(t, x)+c_{x}\left[{ }_{-1} \mathcal{D}_{x}^{2 \mu} u(t, x)\right]=f(t, x), \quad(t, x) \in[0, T] \times[-1,1],(70)
$$

subject to $u(x, 0)=0$ and $u(-1, t)=0$ when $\tau, \mu \in(0,1 / 2]$. In this case, the FPDE (70) appears as Time- and Space-Fractional Advection (TSFA) equation, where we set $c_{x}=1$. We then seek the solution to (70) in terms of a linear combination of elements in $U_{N}$, now consisting of only two dimensions, i.e., time $t$ and space $x$, of the form

$$
u_{N}(t, x)=\sum_{n=\lceil 2 \tau\rceil}^{\mathcal{N}} \sum_{m=\lceil 2 \mu\rceil}^{\mathcal{M}} \hat{u}_{n, m}\left(\psi_{n}^{\tau} \circ \eta\right)(t)\left(\phi_{m}^{\mu} \circ \xi\right)(x) .
$$

Next, we obtain the corresponding linear system of the Lyapunov equation after carrying out the Kronecker product as

$$
S_{\tau} \mathcal{U} M_{\mu}+M_{\tau} \mathcal{U} S_{\mu}=F
$$

where we represent the unknown coefficient matrix $\mathcal{U}$ in terms of the spatial and temporal eigenvectors as

$$
\mathcal{U}=\sum_{q=\lceil 2 \tau\rceil}^{\mathcal{N}} \sum_{p=\lceil 2 \mu\rceil}^{\mathcal{M}} \kappa_{q, p} \vec{e}_{q}^{\tau} \vec{e}_{p}^{\mu^{T}}
$$


for which $\kappa_{q, p}$ is followed by (59) setting $\gamma=0$ as

$$
\kappa_{q, p}=\frac{\vec{e}_{q}^{\tau^{T}} F \vec{e}_{p}^{\mu}}{\left(\vec{e}_{q}^{\tau^{T}} S_{\tau} \vec{e}_{q}^{\tau}\right) \cdot\left(\vec{e}_{p}^{\mu^{T}} S_{\mu} \vec{e}_{p}^{\mu}\right) \cdot\left(c_{1} \lambda_{q}^{\tau}+\lambda_{p}^{\mu}\right)} .
$$

In Fig. 1, we examine the TSFA problem (70) and study the $p$-refinement in both the temporal (left) and the spatial (right) dimensions. To demonstrate the spectral convergence of the PG spectral method, we plot the log$\log L^{2}$-error versus temporal and spatial expansion orders $\mathcal{N}, \mathcal{M}$. In the temporal $p$-refinement $\tau=1 / 20$ and $9 / 20$ while $\mu=1 / 4$; also in the spatial $p$-refinement, the spatial orders $\mu=1 / 20$ and $9 / 20$ while $\tau=1 / 4$. In this test, we set the simulation time to $T=1$, while the exact solution is $u^{e x t}(x, t)=t^{6+2 / 7}(1+x)^{6+3 / 4}$.

\subsection{Parabolic FPDEs}

First, we consider the following parabolic Time- and Space- Fractional Diffusion (TAFD) equation

$$
\begin{aligned}
{ }_{0} \mathcal{D}_{t}^{2 \tau} u(x, t) & =K_{-1} \mathcal{D}_{x}^{2 \mu} u(x, t)+f(x, t), \quad(x, t) \in[0, T] \times[-1,1] \\
u(x, 0) & =0 \\
u( \pm 1, t) & =0
\end{aligned}
$$

where $\tau \in(0,1 / 2], \mu \in(1 / 2,1), K>0$, which is a well-known model for anomalous sub-diffusion process. In this case, we seek the solution also of the form (71), and obtain a similar linear system as in (72), and hence we obtain

$$
\kappa_{q, p}=\frac{\vec{e}_{q}^{\tau^{T}} F \vec{e}_{p}^{\mu}}{\left(\vec{e}_{q}^{\tau^{T}} S_{\tau} \vec{e}_{q}^{\tau}\right) \cdot\left(\vec{e}_{p}^{\mu^{T}} S_{\mu} \vec{e}_{p}^{x}\right) \cdot\left(\lambda_{p}^{\mu}-K \lambda_{q}^{\tau}\right)} .
$$

for $p=\lceil 2 \tau\rceil, \cdots, \mathcal{N}$ and $q=\lceil 2 \mu\rceil, \cdots, \mathcal{M}$. In Fig. 2, we solve the TSFD problem (75) and study the $p$-refinement in the spacial dimension. We plot the $\log$ - $\log L^{2}$-error versus the spatial expansion order $\mathcal{M}$. Similarly, in the spatial $p$-refinement, the spatial orders $\mu=11 / 20$ and $19 / 20$ while $\tau=1 / 4$. In both cases, the spectral convergence of the solution is achieved. Since, the exact solution is fixed in each case, the convergence rate corresponding to $\mu=11 / 20$ appears to be larger than the case where $\mu=19 / 20$ as expected. It is naturally due to the higher regularity requirement in the weak form corresponding to the second test-case. In the temporal $p$-refinement similar results were observed as in Fig.1 (left). 


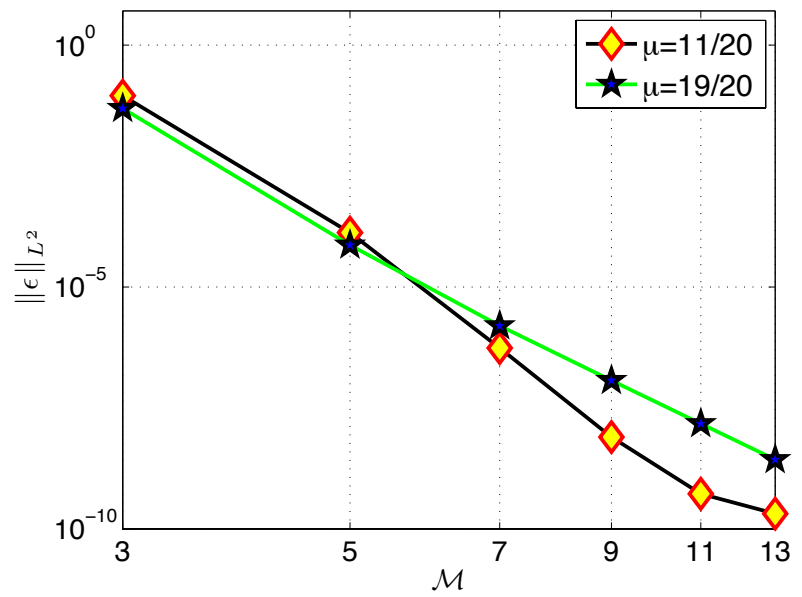

Figure 2: TSFD, spatial p-refinement: $\log -\log L^{2}$-error versus spatial expansion orders $M$. Here, the spatial orders $\mu=11 / 20$ and $19 / 20$ while $\tau=1 / 4$, and the exact solution is $u^{e x t}(x, t)=t^{6+2 / 7}\left[(1+x)^{6+3 / 4}-2^{38 / 35}(1+x)^{5+1 / 2}\right]$.

\subsection{Elliptic FPDEs}

We examine the well-known elliptic Helmholtz/Poisson equations, rendered fractional in two-dimensional (in space) domains. We choose the spatial computational domain as $\Omega=[-1,1] \times[-1,1]$, and consider the following problem

$$
\begin{aligned}
{ }_{-1} \mathcal{D}_{x_{1}}^{2 \mu_{1}} u\left(x_{1}, x_{2}\right)+{ }_{-1} \mathcal{D}_{x_{2}}^{2 \mu_{2}} u\left(x_{1}, x_{2}\right)+\gamma u\left(x_{1}, x_{2}\right) & =f\left(x_{1}, x_{2}\right), \quad \text { in } \Omega(77) \\
u\left(x_{1}, x_{2}\right) & =0, \quad \text { on } \partial \Omega
\end{aligned}
$$

where $\gamma>0, \mu_{1}, \mu_{2} \in(1 / 2,1)$, which reduces to the Space-Fractional Poisson equation when $\gamma=0$. Here, we present a general scheme in addition to a linear fast solver for both problems.

We then seek the solution to (77) in terms of a linear combination of elements in $U_{N}$ in absence of the time-basis, consisting of only two dimensions of the form

$$
u_{N}\left(x_{1}, x_{2}\right)=\sum_{m_{1}=2}^{\mathcal{M}_{1}} \sum_{m_{2}=2}^{\mathcal{M}_{2}} \hat{u}_{m_{1}, m_{2}}\left(\phi_{m_{1}}^{\mu_{1}} \circ \xi_{1}\right)\left(x_{1}\right)\left(\phi_{m_{2}}^{\mu_{2}} \circ \xi_{2}\right)\left(x_{2}\right),
$$

for which we represent the unknown coefficient matrix $\mathcal{U}$ in terms of the 


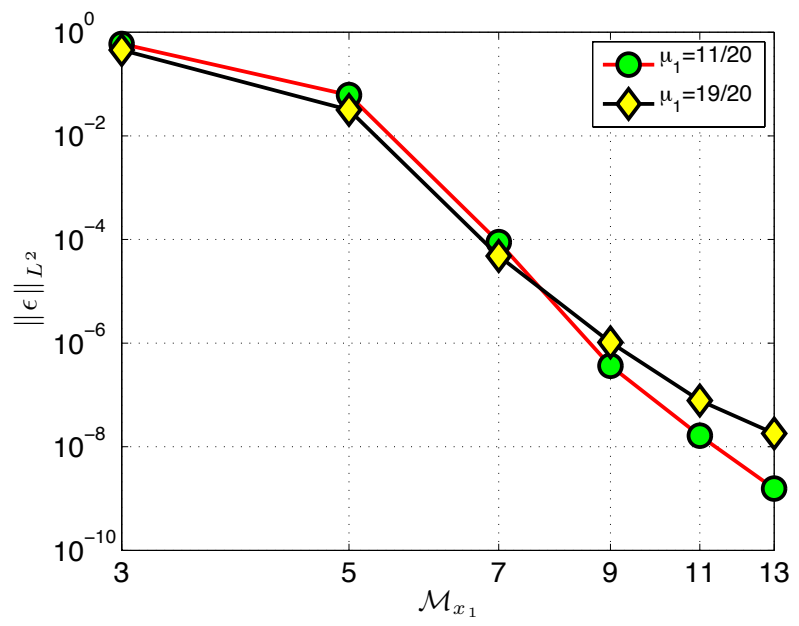

Figure 3: Space-fractional Helmholtz problem with $\gamma=1$, spatial p-refinement in $\mathrm{x}$ dimension: $\log$ - $\log L^{2}$-error versus spatial expansion orders $\mathcal{M}_{x_{1}}$. Here, the spatial orders are $\mu_{1}=11 / 20$ and $19 / 20$ while $\mu_{2}=15 / 20$, is kept constant. The exact solution is $u^{e x t}\left(x_{1}, x_{2}\right)=\left[\left(1+x_{1}\right)^{6+3 / 4}-2^{5 / 4}\left(1+x_{1}\right)^{5+1 / 2}\right]\left[\left(1+x_{2}\right)^{6+4 / 9}-2^{73 / 63}\left(1+x_{2}\right)^{5+2 / 7}\right]$. A similar convergence curve is achieved in the $p$-refinement performed in the $\mathrm{y}$-dimension, also for the case of $\gamma=0$.

spatial eigenvectors as

$$
\mathcal{U}=\sum_{p_{1}=2}^{\mathcal{M}_{1}} \sum_{p_{2}=2}^{\mathcal{M}_{2}} \kappa_{p_{1}, p_{2}} \vec{e}_{p_{1}}^{\mu_{1}} \vec{e}_{p_{2}}^{\mu_{2}{ }^{T}}
$$

where

$$
\kappa_{p_{1}, p_{2}}=\frac{\vec{e}_{p_{1}}^{\mu_{1}^{T}} G \vec{e}_{p_{2}}^{\mu_{2}}}{\left(\vec{e}_{p_{1}}^{\mu_{1} T} S_{\mu_{1}} \vec{e}_{p_{1}}^{\mu_{1}}\right) \cdot\left(\vec{e}_{p_{2}}^{\mu_{2} T} S_{\mu_{2}} \vec{e}_{p_{2}}^{\mu_{2}}\right) \cdot\left(\lambda_{p_{1}}^{\mu_{1}}+\lambda_{p_{2}}^{\mu_{2}}+\gamma \lambda_{p_{1}}^{\mu_{1}} \lambda_{p_{2}}^{x_{2}}\right)} .
$$

In Fig. 3, we solve the fractional Helmholzt problem (77) and study the $p$-refinement in the spacial $x_{1}$-dimension. To demonstrate the spectral convergence of the fast FPDE solver, we plot the log-log $L^{2}$-error versus the spatial expansion order $\mathcal{M}_{x_{1}}$. The spatial orders $\mu_{1}=11 / 20$ and $19 / 20$ while $\mu_{2}=15 / 20$. Similar to the spatial $p$-convergence in Fig.2, the convergence rate corresponding to $\mu_{1}=11 / 20$ appears to be larger than the case where $\mu_{2}=19 / 20$. We observe a similar $p$-refinement in the $x_{2}$-dimension as well. 


\subsection{Higher-Dimensional FPDEs}

Next, we employ our PG method and the fast solver in even higher dimensional problems to exhibit the generality and efficiency of the scheme. In Table 1, the convergence results and CPU time of the unified PG spectral method in higher-dimensional problems are examined. Particularly, we employ this scheme to solve the time- and space- fractional advection equation (TSFA)

$$
{ }_{0} \mathcal{D}_{t}^{2 \tau} u+{ }_{-1} \mathcal{D}_{x_{1}}^{2 \mu_{1}} u+{ }_{-1} \mathcal{D}_{x_{2}}^{2 \mu_{2}} u+\cdots+{ }_{-1} \mathcal{D}_{x_{d}}^{2 \mu_{d}} u=f,
$$

where $2 \tau=2 \mu_{j}=1 / 2$, subject to homogeneous Dirichlet boundary conditions in a four-dimensional (4-D), six-dimensional (6-D), and ten-dimensional (10-D) space-time hypercube domains. The error is measured by the essential norm $\|\epsilon\|_{L^{\infty}}=\left\|u-u^{e x t}\right\|_{L^{\infty}} /\left\|u^{e x t}\right\|_{L^{\infty}}$, which is stronger than the $L^{2}$-norm and is normalized by the essential norm of the exact solution $u^{e x t}(t, \vec{x})=$ $\left[t \prod_{j=1}^{d}\left(1+x_{j}\right)\right]^{2+2 / 5}$ for the sake of consistency. The CPU time (seconds) is measured on a single core Intel (Xeon X5550) $2.67 \mathrm{GHz}$ processor. In each step of the $p$-refinement, we uniformly increase the bases order by one in all dimensions. All the computations are performed in Mathematica 8. These simulations highlight that the unified PG spectral method is efficient even for a $10-\mathrm{D}$ problem run on a $\mathrm{PC}$ in less than an hour!

\subsection{Time-integration when $2 \tau=1$}

We recall that our unified PG spectral method works equally well when the temporal time-derivative order $2 \tau=1$. In general, a first-order in time PDE/FPDE reads

$$
\frac{\partial u}{\partial t}=F\left(u ; t, x_{1}, \cdots, x_{d}\right),
$$

where the operator $F\left(u ; t, x_{1}, \cdots, x_{d}\right)$ is given as

$$
F\left(u ; t, x_{1}, \cdots, x_{d}\right)=f\left(t, x_{1}, \cdots, x_{d}\right)-\sum_{j=1}^{d} c_{j}\left[{ }_{a_{j}} \mathcal{D}_{x_{j}}^{2 \mu_{j}} u\right]+\gamma u,
$$

in view of (8). Here, we regard the PG method as an alternative scheme for spectrally accurate time-integration for a general $F\left(u ; t, x_{1}, \cdots, x_{d}\right)$, rather than utilizing existing algebraically accurate methods, including multi-step methods such as the Adams family and stiffly-stable schemes, also multi-stage approaches such as the Runge-Kutta method. 
Table 1: Convergence study and CPU time of the unified PG spectral method employed in the time- and space- fractional advection equation (TSFA) ${ }_{0} \mathcal{D}_{t}^{2 \tau} u+\sum_{j=1}^{d}\left[{ }_{-1} \mathcal{D}_{x_{j}}^{2 \mu_{j}} u\right]=f$, where $2 \tau=2 \mu_{j}=1 / 2, j=1,2, \cdots, d$, subject to homogeneous Dirichlet boundary conditions in four-dimensional (4-D), six-dimensional (6-D), and ten-dimensional (10-D) space-time hypercube domains, where $D=1+d$. The error is measured by the essential norm $\|\epsilon\|_{L^{\infty}}=\left\|u-u^{e x t}\right\|_{L^{\infty}} /\left\|u^{e x t}\right\|_{L^{\infty}}$, which is normalized by the essential norm of the exact solution $u^{\text {ext }}(t, \vec{x})=\left[t \prod_{j=1}^{d}\left(1+x_{j}\right)\right]^{2+2 / 5}$, where $t \in[0,1]$ and $x \in[-1,1]^{d}$. The CPU time (seconds) is obtained on a Intel (Xeon X5550) 2.67GHz processor. In each step, we uniformly increase the bases order by one in all dimensions.

\section{4-D TSFA}

\begin{tabular}{ccc}
\hline \hline $\mathcal{N}=\mathcal{M}_{1}=\cdots=\mathcal{M}_{d}$ & $\|\epsilon\|_{L^{\infty}}$ & CPU Time (seconds) \\
\hline 2 & 0.576869 & 0.006333 \\
\hline 3 & 0.034706 & 0.015997 \\
\hline 4 & 0.003990 & 0.041994 \\
\hline 5 & 0.0009071 & 0.105984 \\
\hline
\end{tabular}

6-D TSFA

\begin{tabular}{ccc}
\hline \hline $\mathcal{N}=\mathcal{M}_{1}=\cdots=\mathcal{M}_{d}$ & $\|\epsilon\|_{L^{\infty}}$ & CPU Time (seconds) \\
\hline 2 & 0.741056 & 0.014748 \\
\hline 3 & 0.055171 & 0.134313 \\
\hline 4 & 0.006578 & 0.821208 \\
\hline 5 & 0.001525 & 3.546791 \\
\hline
\end{tabular}

10-D TSFA

\begin{tabular}{ccc}
\hline \hline $\mathcal{N}=\mathcal{M}_{1}=\cdots=\mathcal{M}_{d}$ & $\|\epsilon\|_{L^{\infty}}$ & CPU Time (seconds) \\
\hline 2 & 0.903357 & 0.288956 \\
\hline 3 & 0.095305 & 18.45320 \\
\hline 4 & 0.0119229 & $370.1(\approx 6 \mathrm{~min})$ \\
\hline 5 & 0.0028102 & $3332.4(\approx 55 \mathrm{~min})$ \\
\hline
\end{tabular}


The idea of employing the PG spectral method when $2 \tau=1$ is simply based on the useful property (5) by which a full first-order derivative $d / d t$ can be decomposed into a product of the sequential $\left(\frac{1}{2}\right)$-th order derivatives ${ }_{0} \mathcal{D}_{t}^{1 / 2}{ }_{0} \mathcal{D}_{t}^{1 / 2}$, a result that is not valid in the standard (integer-order) calculus. Hence, by virtue of the fractional integration-by-parts, such a decomposition artificially induces non-locality to the temporal term in the corresponding weak form. Therefore, it provides an appropriate framework for global (spectral) treatment of the temporal term using our unified PG spectral method. To this end, we carry out the time-integration when $2 \tau=1$ in the following FPDE

$$
\partial u / \partial t+\sum_{j=1}^{3}{ }_{-1} \mathcal{D}_{x_{j}}^{2 \mu_{j}} u=f
$$

in $\Omega \subset \mathbb{R}^{1+3}$, where in general $\mu_{j} \in(0,1)$. Here, we set $\mu_{j}=1 / 2$ for simplicity, which recovers the standard time-dependent advection equation in three-dimensional spatial domain.

In Table 2, we again measure the error by the normalized essential norm, where the exact solution is $u^{e x t}(t, \vec{x})=\left[t \prod_{j=1}^{3}\left(1+x_{j}\right)\right]^{6+2 / 5}$, where $t \in[0,1]$ and $x_{j} \in[-1,1], j=1,2,3$. Similar to the previous case, the CPU time (seconds) is obtained on a single-core Intel (Xeon X5550) 2.67GHz processor, where we uniformly increase the bases order by one in all dimensions in each step. In these simulations, we globally treat the time-axis in addition to other spatial dimensions. The CPU time and the spectral convergence strongly highlights the efficiency of our approach, where a 4-D problem (i.e., 1-D in time and 3-D in space) can be highly accurately solved in a fraction of minute!

\section{Summary and Discussion}

We developed a unified and spectrally accurate Petrov-Galerkin (PG) spectral method for a weak formulation of the general linear FPDE of the form ${ }_{0} \mathcal{D}_{t}^{2 \tau} u+\sum_{j=1}^{d} c_{x_{j}}\left[{ }_{a_{j}} \mathcal{D}_{x_{j}}^{2 \mu_{j}} u\right]+\gamma u=f, \tau, \mu_{j} \in(0,1)$, in a $(1+d)$ dimensional space-time domain subject to Dirichlet initial and boundary conditions. We demonstrated that this scheme performs well for the whole family of linear hyperbolic-, parabolic- and elliptic-like equations with the same ease. We developed our PG method based on a new spectral theory for fractional Sturm-Liouville problems (FSLPs), recently introduced in [1]. 
Table 2: Time-Integration when $2 \tau=1: \partial u / \partial t+\sum_{j=1}^{3}\left[{ }_{-1} \mathcal{D}_{x_{j}}^{2 \mu_{j}} u\right]=f$ in $\Omega \subset \mathbb{R}^{1+3}$, where $t \in[0,1]$ and $x_{j} \in[-1,1], j=1,2,3$. Here, we set $\mu_{j}=1 / 2$ to fully recover the standard time-dependent advection equation in three-dimensional spatial domain. However, in general $\mu_{j} \in(0,1)$. The error is measured by the essential norm $\|\epsilon\|_{L^{\infty}}=\left\|u-u^{e x t}\right\|_{L^{\infty}} /\left\|u^{e x t}\right\|_{L^{\infty}}$, which is normalized by the essential norm of the exact solution is $u^{e x t}(t, \vec{x})=\left[t \prod_{j=1}^{3}\left(1+x_{j}\right)\right]^{6+2 / 5}$. The CPU time (seconds) is obtained on a Intel (Xeon X5550) 2.67GHz processor. In each step, we uniformly increase the bases order by one in all dimensions.

Integer-Order Time-Integration

\begin{tabular}{ccc}
\hline \hline $\mathcal{N}=\mathcal{M}_{1}=\mathcal{M}_{2}=\mathcal{M}_{3}$ & $\|\epsilon\|_{L^{\infty}}$ & CPU Time (seconds) \\
\hline 3 & 0.6225970 & 0.051992 \\
\hline 5 & 0.0336570 & 0.352947 \\
\hline 7 & $1.34 \times 10^{-5}$ & 1.9737 \\
\hline 9 & $1.06 \times 10^{-7}$ & 3.7894 \\
\hline 11 & $3.52 \times 10^{-9}$ & 9.7365 \\
\hline 13 & $2.54 \times 10^{-10}$ & 21.472 \\
\hline
\end{tabular}


In the present method, all the aforementioned matrices are constructed $e x$ actly and efficiently. We additionally performed the stability analysis (in 1-D) and the corresponding convergence study of the scheme (in multi-D). Moreover, we formulated a new general fast linear solver based on the eigenpairs of the corresponding temporal and spatial mass matrices with respect to the stiffness matrices, which significantly reduces the computational cost in higher-dimensional problems e.g., $(1+3),(1+5)$ and $(1+9)$-dimensional FPDEs.

In the $p$-refinement tests performed in the aforementioned problems, we kept the fractional order to be the middle-value (either $1 / 2$ or $3 / 2$ ) in the fixed direction, and we examined some limit fractional orders in the other direction. However, we numerically observe that if the fixed fractional order is taken to be closer to the limit values (i.e., either 0 or 1 ), the mode of spectral convergence remains unchanged but we achieve a different rate of convergence to be verified in our future theoretical analysis.

Alternating Direction Implicit (ADI) methods (see e.g., [49]) are another way of solving space-fractional FPDEs in higher dimensional problems. In this approach, a one-dimensional space-fractional FPDE solver with a loworder (finite-difference) time integrator can be employed to solve 2-D or 3-D problems. However, we note that ADI naturally cannot treat time- and space-fractional FPDEs. Moreover, the temporal rate of convergence in this approach is algebraic in contrast to the high accuracy in the spatial discretizations. Hence, the computational complexity of this approach becomes exceedingly large in higher-dimensional FPDEs.

In practice, the enforcement of periodic boundary conditions to FPDEs is not possible since it is not clear how to define history (memory) for a periodic function. Moreover, we note that Riemann-Liouville fractional derivatives in time/space only allow us to impose homogeneous initial/boundary conditions to the corresponding FPDEs to be wellposed. However, we note that our PG spectral method is also applicable in such problems in the following manner. When inhomogeneous Dirichlet conditions are enforced, the corresponding derivatives are usually replaced by Caputo fractional derivatives. We illustrate such a treatment in the following model problem posed subject to an inhomogeneous initial condition:

$$
\begin{aligned}
{ }_{0}^{C} \mathcal{D}_{t}^{2 \tau} u & ={ }_{-1} \mathcal{D}_{x}^{2 \mu} u+f(x, t), \\
u(x, 0) & =g(x), \\
u( \pm 1, t) & =0
\end{aligned}
$$


in which $2 \mu \in(1,2), g(x) \in C^{0}([-1,1])$, and ${ }_{0}^{C} \mathcal{D}_{t}^{2 \tau}(\cdot)$ denotes the Caputo fractional derivative of order $2 \tau \in(0,1)$, which is defined via interchanging the order of differentiation and integration in (3), see e.g., [3]. Now, we define $U(x, t)=u(x, t)-g(x)$, and taking into account that ${ }_{0}^{C} \mathcal{D}_{t}^{2 \tau} g(x) \equiv 0$. Then, by substituting $u=U+g$ into (82) and noting that ${ }_{0}^{C} \mathcal{D}_{t}^{2 \tau} U={ }_{0} \mathcal{D}_{t}^{2 \tau} U$ due to the homogeneity of $U(x, 0)$, we obtain the transformed problem as

$$
\begin{aligned}
{ }_{0} \mathcal{D}_{t}^{2 \tau} U & ={ }_{-1} \mathcal{D}_{x}^{2 \mu} U+\tilde{f}(x, t), \\
U(x, 0) & =0 \\
U( \pm 1, t) & =0
\end{aligned}
$$

in which $\tilde{f}(x, t)=f(x, t)+{ }_{-1} \mathcal{D}_{x}^{2 \mu} g(x)$. Therefore, we can treat such inhomogeneous conditions by our unified PG spectral method through homogenizing the problem and modifying the forcing term on the right-hand side. The same approach applies to inhomogeneous boundary conditions.

Although the proposed unified PG method enjoys the high accuracy of the discretization in time and space in addition to its efficiency in solving higher-dimensional problems, treating FPDEs in complex geometries still remains a great challenge to be addressed in our future works. Moreover, special care should be taken when the FPDE of the interest is associated with variable coefficients and/or non-linearity. In [44], we have employed the fractional bases to construct a new class of fractional Lagrange interpolants i.e., fractional nodal rather than modal basis functions presented here, to develop efficient and spectrally accurate collocation methods for a variety of FODEs and FPDEs including non-linear space-fractional Burgers' equation.

\section{Acknowledgements}

This work was supported by the Collaboratory on Mathematics for Mesoscopic Modeling of Materials (CM4) at PNNL funded by the Department of Energy, by OSD/MURI and by NSF/DMS. 


\section{Appendix}

Theorem 6.1. The temporal stiffness matrix $S_{\tau}$ corresponding to the timefractional order $\tau \in(0,1)$ is a diagonal $\mathcal{N} \times \mathcal{N}$ matrix, whose entries are explicitly given as

$$
\left\{S_{\tau}\right\}_{n, n}=\widetilde{\sigma}_{n} \sigma_{n}\left[\frac{\Gamma(n+\tau)}{\Gamma(n)}\right]^{2}\left(\frac{2}{T}\right)^{2 \tau-1} \frac{2}{2 n-1}, \quad n=1,2, \cdots, \mathcal{N} .
$$

Proof. By the PG projection, the $(r, n)$-th entry of the stiffness matrix, $r, n=$ $1,2, \cdots, \mathcal{N}$, is defined as

$$
\left\{S_{\tau}\right\}_{r, n}=\int_{0}^{T}{ }_{t} \mathcal{D}_{T}^{\tau}\left(\Psi_{r}^{\tau} \circ \eta\right)(t){ }_{0} \mathcal{D}_{t}^{\tau}\left(\psi_{n}^{\tau} \circ \eta\right)(t) d t,
$$

Following [1], we obtain the Riemann-Liouville left-sided time-fractional derivative of the temporal basis as

$$
{ }_{0} \mathcal{D}_{t}^{\tau}\left(\psi_{n}^{\tau} \circ \eta\right)(t)=\sigma_{n}\left(\frac{2}{T}\right)^{\tau} \frac{\Gamma(n+\tau)}{\Gamma(n)} P_{n-1}(2 t / T-1),
$$

where $P_{n-1}(2 t / T-1)$ represents the $(n-1)$-th order Legendre polynomial in $t \in[0, T]$. Also, we obtain the right-sided time-fractional derivative of the temporal basis again following [1] as

$$
{ }_{t} \mathcal{D}_{T}^{\tau}\left(\Psi_{r}^{\tau} \circ \eta\right)(t)=\widetilde{\sigma}_{r}\left(\frac{2}{T}\right)^{\tau} \frac{\Gamma(r+\tau)}{\Gamma(r)} P_{r-1}(2 t / T-1) .
$$

Now, by plugging (85) and (86) into (84), we obtain

$$
\begin{aligned}
\left\{S_{\tau}\right\}_{r, n} & =\tilde{\sigma}_{r} \sigma_{n} \frac{\Gamma(r+\tau)}{\Gamma(r)} \frac{\Gamma(n+\tau)}{\Gamma(n)}\left(\frac{2}{T}\right)^{2 \tau} \int_{0}^{T} P_{r-1}(x(t)) P_{n-1}(x(t)) d(87) \\
& =\widetilde{\sigma}_{r} \sigma_{n} \frac{\Gamma(r+\tau)}{\Gamma(r)} \frac{\Gamma(n+\tau)}{\Gamma(n)}\left(\frac{2}{T}\right)^{2 \tau-1} \int_{-1}^{1} P_{r-1}(x) P_{n-1}(x) d x \\
& =\tilde{\sigma}_{r} \sigma_{n} \frac{\Gamma(r+\tau)}{\Gamma(r)} \frac{\Gamma(n+\tau)}{\Gamma(n)}\left(\frac{2}{T}\right)^{2 \tau-1} \frac{2}{2 n-1} \delta_{r n},
\end{aligned}
$$

by the orthogonality of the Legendre polynomials, where $\delta_{r n}$ is the Kronecker delta functions. 
Theorem 6.2. (I) If $\mu_{j} \in(0,1 / 2]$, the spatial stiffness matrix $S_{\mu_{j}}$ is a diagonal $\mathcal{M}_{j} \times \mathcal{M}_{j}$ matrix, whose entries are explicitly given as

$$
\left\{S_{\mu_{j}}\right\}_{k, k}=\widetilde{\sigma}_{k} \sigma_{k}\left[\frac{\Gamma\left(k+\mu_{j}\right)}{\Gamma(k)}\right]^{2}\left(\frac{2}{L_{j}}\right)^{2 \mu_{j}-1} \frac{2}{2 k-1}, \quad k=1,2, \cdots, \mathcal{M}_{j} .
$$

(II) If $\mu_{j} \in(1 / 2,1), S_{\mu_{j}}$ is a symmetric tridiagonal $\left(\mathcal{M}_{j}-1\right) \times\left(\mathcal{M}_{j}-1\right)$ with entries, explicitly given as

$$
\begin{array}{r}
\left\{S_{\mu_{j}}\right\}_{k, m}=b_{k} a_{m}\left[\frac{\Gamma\left(k+\mu_{j}\right)}{\Gamma(k)}\right]^{2}\left(\frac{2}{L_{j}}\right)^{2 \mu_{j}-1} \frac{2}{2 k-1}\left(\delta_{k, m}-\epsilon_{m}^{\mu_{j}} \delta_{k, m-1}\right)+ \\
\epsilon_{k}^{\mu_{j}} b_{k} a_{m}\left[\frac{\Gamma\left(k-1+\mu_{j}\right)}{\Gamma(k-1)}\right]^{2}\left(\frac{2}{L_{j}}\right)^{2 \mu_{j}-1} \frac{2}{2 k-3}\left(\delta_{k-1, m}-\epsilon_{m}^{\mu_{j}} \delta_{k-1, m-1}\right),
\end{array}
$$

$k, m=2,3, \cdots, \mathcal{M}_{j}$ and $L_{j}=b_{j}-a_{j}$.

Proof. The first part, when $\mu_{j} \in(0,1 / 2]$ is similar to the proof in Thm. 6.1, however, carried out on the interval $\left[a_{j}, b_{j}\right]$ rather than $[0, T]$. Here, the $\mu_{j}$-th order left-sided Riemann-Liouville fractional derivative of $\left(\phi_{m_{j}}^{\mu_{j}} \circ \xi_{j}\right)\left(x_{j}\right)$ is given following [1] as

$$
{ }_{a_{j}} \mathcal{D}_{x_{j}}^{\mu_{j}}\left(\phi_{m}^{\mu_{j}} \circ \xi_{j}\right)\left(x_{j}\right)= \begin{cases}B_{m}^{\mu_{j}} P_{m-1}\left(\xi\left(x_{j}\right)\right), & \mu_{j} \in(0,1 / 2], \\ B_{m}^{\mu_{j}} P_{m-1}\left(\xi\left(x_{j}\right)\right)-C_{m}^{\mu_{j}} P_{m-2}\left(\xi\left(x_{j}\right)\right), & \mu_{j} \in(1 / 2,1),\end{cases}
$$

for $m=\left\lceil 2 \mu_{j}\right\rceil, \cdots, \mathcal{M}_{j}$ in the $j$-th spatial dimension, where the coefficient $B_{m_{j}}^{\mu_{j}}=\sigma_{m}\left(2 / L_{j}\right)^{2 \mu_{j}} \Gamma\left(m+\mu_{j}\right) / \Gamma(m)$ and $C_{m}^{\mu_{j}}=\sigma_{m}\left(2 / L_{j}\right)^{2 \mu_{j}} \epsilon_{m}^{\mu_{j}} \Gamma\left(m-1+\mu_{j}\right) / \Gamma(m-1)$; in addition, the $\mu_{j}$-th order left-sided Riemann-Liouville fractional derivative of $\left(\Phi_{m}^{\mu_{j}} \circ \xi_{j}\right)\left(x_{j}\right)$ is obtained as

$$
{ }_{x_{j}} \mathcal{D}_{b_{j}}^{\mu_{j}}\left(\Phi_{m}^{\mu_{j}} \circ \xi_{j}\right)\left(x_{j}\right)= \begin{cases}\mathcal{B}_{k}^{\mu_{j}} P_{k-1}\left(\xi\left(x_{j}\right)\right), & \mu_{j} \in(0,1 / 2], \\ \mathcal{B}_{k}^{\mu_{j}} P_{k-1}\left(\xi\left(x_{j}\right)\right)-\mathcal{C}_{k}^{\mu_{j}} P_{k-2}\left(\xi\left(x_{j}\right)\right), & \mu_{j} \in(1 / 2,1),\end{cases}
$$

for $k=\left\lceil 2 \mu_{j}\right\rceil, \cdots, \mathcal{M}_{j}$ in the $j$-th spatial dimension, in which we set the coefficient $\mathcal{B}_{k}^{\mu_{j}}=\widetilde{\sigma}_{k}\left(2 / L_{j}\right)^{2 \mu_{j}} \Gamma\left(k+\mu_{j}\right) / \Gamma(k)$ and $\mathcal{C}_{k}^{\mu_{j}}=\widetilde{\sigma}_{k}\left(2 / L_{j}\right)^{2 \mu_{j}} \epsilon_{k}^{\mu_{j}} \Gamma\left(k-1+\mu_{j}\right) / \Gamma(k-1)$. 
For the second part, when $\mu_{j} \in(1 / 2,1)$, the $(k, m)$-th entry of $S_{\mu_{j}}$ is

$$
\left\{S_{\mu_{j}}\right\}_{k, m}=\int_{a_{j}}^{b_{j}}{ }_{x_{j}} \mathcal{D}_{b_{j}}^{\mu_{j}}\left(\Phi_{k}^{\mu_{j}} \circ \xi_{j}\right)\left(x_{j}\right)_{a_{j}} \mathcal{D}_{x_{j}}^{\mu_{j}}\left(\phi_{m}^{\mu_{j}} \circ \xi_{j}\right)\left(x_{j}\right) d x_{j} .
$$

Next, by virtue of (88) and (89), also by an affine mapping from $\left[a_{j}, b_{j}\right]$ to the standard interval $[-1,1]$, we obtain

$$
\begin{aligned}
\left\{S_{\mu_{j}}\right\}_{k, m}=\left(\frac{L_{j}}{2}\right) \int_{-1}^{1} & {\left[B_{m}^{\mu_{j}} P_{m-1}\left(\xi_{j}\right)-C_{m}^{\mu_{j}} P_{m-2}\left(\xi_{j}\right)\right] } \\
& {\left[\mathcal{B}_{k}^{\mu_{j}} P_{k-1}\left(\xi_{j}\right)-\mathcal{C}_{k}^{\mu_{j}} P_{k-2}\left(\xi_{j}\right)\right] d \xi_{j} . }
\end{aligned}
$$

Hence, by the orthogonality of the Legendre polynomials we obtain

$$
\begin{aligned}
\left\{S_{\mu_{j}}\right\}_{k, m}=\left(\frac{L_{j}}{2}\right) & {\left[B_{m}^{\mu_{j}} \mathcal{B}_{k}^{\mu_{j}} \frac{2}{2 k-1} \delta_{k, m}\right.} \\
& -C_{m}^{\mu_{j}} \mathcal{B}_{k}^{\mu_{j}} \frac{2}{2 k-1} \delta_{k, m-1} \\
& +B_{m}^{\mu_{j}} \mathcal{C}_{k}^{\mu_{j}} \frac{2}{2 k-3} \delta_{k-1, m} \\
& \left.-C_{m}^{\mu_{j}} \mathcal{C}_{k}^{\mu_{j}} \frac{2}{2 k-3} \delta_{k-1, m-1}\right]
\end{aligned}
$$

which completes the proof, while the symmetry of the stiffness matrix can be easily checked.

Theorem 6.3. The temporal and the spatial mass matrices $M_{\tau}$ as well as $M_{\mu_{j}}$ are symmetric. Moreover, their entries can be computed exactly by employing a Gauss-Lobatto-Jacobi (GLJ) rule with respect to the weight function $(1-\xi)^{\alpha}(1+\xi)^{\alpha}, \xi \in[-1,1]$, where $\alpha=\tau / 2$ in the temporal and $\alpha=\mu_{j}$ for the spatial case.

Proof. The entries of $M_{\tau}$ in our PG spectral method are defined as

$$
\left\{M_{\tau}\right\}_{r, n}=\int_{0}^{T}\left(\Psi_{r}^{\tau} \circ \eta\right)(t)\left(\psi_{m}^{\mu_{t}} \circ \eta\right)(t) d t
$$


which be computed exactly as

$$
\begin{aligned}
\left\{M_{\tau}\right\}_{r, n} & =\widetilde{\sigma}_{r} \sigma_{n}\left(\frac{2}{T}\right)^{\tau} \int_{0}^{T} t^{\tau}(T-t)^{\tau} P_{r-1}^{\tau,-\tau}(\eta(t)) P_{n-1}^{-\tau, \tau}(\eta(t)) d t \\
& =\widetilde{\sigma}_{r} \sigma_{n} \frac{T}{2} \int_{-1}^{1}(1-\eta)^{\tau}(1+\eta)^{\tau} P_{r-1}^{\tau,-\tau}(\eta) P_{n-1}^{-\tau, \tau}(\eta) d \eta \\
& =\widetilde{\sigma}_{r} \sigma_{n} \frac{T}{2} \sum_{q=1}^{\mathcal{Q}} w_{q} P_{r-1}^{\tau,-\tau}\left(\eta_{q}\right) P_{n-1}^{-\tau, \tau}\left(\eta_{q}\right)
\end{aligned}
$$

in which $\mathcal{Q} \geq \mathcal{N}+2$ represents the minimum number of GLJ quadrature points $\left\{\eta_{q}\right\}_{q=1}^{\mathcal{Q}}$, associated with the weigh function $(1-\eta)^{\tau}(1+\eta)^{\tau}$, for exact quadrature, and $\left\{w_{q}\right\}_{q=1}^{Q}$ are the corresponding quadrature weights. From the exact discrete rule, recalling the definition of $\sigma_{n}$ and $\widetilde{\sigma}_{r}$, employing the property of the Jacobi polynomials where $P_{n}^{\alpha, \beta}(-x)=(-1)^{n} P_{n}^{\beta, \alpha}(x)$, moreover, noting that $\left\{\eta_{q}\right\}_{q=1}^{\mathcal{Q}}$ and $\left\{w_{q}\right\}_{q=1}^{Q}$ are symmetric with respect to the reference point, it is easy to show that $\left\{M_{\tau}\right\}_{r, n}=\left\{M_{\tau}\right\}_{n, r}$.

The spatial mass matrix $M_{\mu_{j}}$, when $\mu_{j} \in(0,1 / 2]$, is also $\mathcal{M}_{j} \times \mathcal{M}_{j}$, whose entries are computed similarly as

$$
\left\{M_{\mu_{j}}\right\}_{k, m}=\widetilde{\sigma}_{k} \sigma_{m} \frac{L_{j}}{2} \sum_{q=1}^{Q} w_{q} P_{k-1}^{\mu_{j},-\mu_{j}}\left(\xi_{q}\right) P_{m-1}^{-\mu_{j}, \mu_{j}}\left(\xi_{q}\right)
$$

in which $\mathcal{Q} \geq \mathcal{M}_{j}+2$ represents the minimum number of GLJ quadrature points $\left\{\xi_{q}\right\}_{q=1}^{\mathcal{Q}}$, associated with the weigh function $(1-\xi)^{\mu_{j}}(1+\xi)^{\mu_{j}}$, for exact quadrature. We can also show that $M_{\mu_{j}}$ is symmetric and that the GJL rule is exact when $\mathcal{Q} \geq \mathcal{M}_{j}+2$.

Finally, when $\mu_{j} \in(1 / 2,1)$, the spatial mass matrix $M_{\mu_{j}}$, becomes $\left(\mathcal{M}_{j}-\right.$ 
1) $\times\left(\mathcal{M}_{j}-1\right)$, whose entries are computed exactly as

$$
\begin{aligned}
\left\{M_{\mu_{j}}\right\}_{k, m} & =\int_{a_{j}}^{b_{j}}\left(\Phi_{k}^{\mu_{j} / 2} \circ \xi\right)\left(x_{j}\right)\left(\phi_{m}^{\mu_{j} / 2} \circ \xi\right)\left(x_{j}\right) d x_{j} \\
& =\widetilde{\sigma}_{k_{j}} \sigma_{m_{j}}\left[\int_{-1}^{1}{ }^{(2)} \mathcal{P}_{k_{j}}^{\mu_{j}}\left(\xi\left(x_{j}\right)\right)^{(1)} \mathcal{P}_{m}^{\mu_{x}}\left(\xi\left(x_{j}\right)\right) d x_{j}\right. \\
& -\epsilon_{m}^{\mu_{x}} \int_{-1}^{1}{ }^{(2)} \mathcal{P}_{k_{j}}^{\mu_{j}}\left(\xi\left(x_{j}\right)\right){ }^{(1)} \mathcal{P}_{m_{j}-1}^{\mu_{j}}\left(\xi\left(x_{j}\right)\right) d x_{j} \\
& +\epsilon_{k}^{\mu_{x}} \int_{-1}^{1}{ }^{(2)} \mathcal{P}_{k_{j}-1}^{\mu_{j}}\left(\xi\left(x_{j}\right)\right)^{(1)} \mathcal{P}_{m_{j}}^{\mu_{j}}\left(\xi\left(x_{j}\right)\right) d x_{j} \\
& \left.-\epsilon_{k_{j}}^{\mu_{j}} \epsilon_{m_{j}}^{\mu_{j}} \int_{-1}^{1}{ }^{(2)} \mathcal{P}_{k_{j}-1}^{\mu_{j}}\left(\xi\left(x_{j}\right)\right)^{(1)} \mathcal{P}_{m_{j}-1}^{\mu_{j}}\left(\xi\left(x_{j}\right)\right) d x_{j}\right],
\end{aligned}
$$

where we note that all the above integrations share the same weight function by construction. Hence, we obtain

$$
\begin{aligned}
& \left\{M_{\mu_{j}}\right\}_{k, m}=\widetilde{\sigma}_{k_{j}} \sigma_{m_{j}} \frac{L_{j}}{2} \int_{-1}^{1} \quad(1-\xi)^{\mu_{j}}(1+\xi)^{\mu_{j}} \\
& {\left[P_{k-1}^{\mu_{j},-\mu_{j}}(\xi) P_{m-1}^{-\mu_{j}, \mu_{j}}(\xi)\right.} \\
& \text { - } \epsilon_{m}^{\mu_{x}} P_{k-1}^{\mu_{j},-\mu_{j}}(\xi) P_{m-2}^{-\mu_{j}, \mu_{j}}(\xi) \\
& +\epsilon_{k}^{\mu_{x}} P_{k-2}^{\mu_{j},-\mu_{j}}(\xi) P_{m-1}^{-\mu_{j}, \mu_{j}}(\xi) \\
& \text { - } \left.\quad \epsilon_{k_{j}}^{\mu_{j}} \epsilon_{m_{j}}^{\mu_{j}} \quad P_{k-2}^{\mu_{j},-\mu_{j}}(\xi) \quad P_{m-2}^{-\mu_{j}, \mu_{j}}(\xi)\right] d x_{j}
\end{aligned}
$$

which leads to the following exact GLJ rule

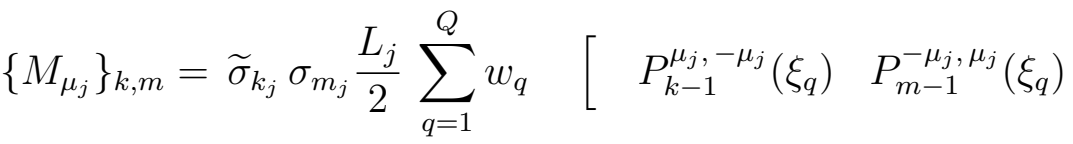

$$
\begin{aligned}
& \text { - } \epsilon_{m}^{\mu_{x}} P_{k-1}^{\mu_{j},-\mu_{j}}\left(\xi_{q}\right) P_{m-2}^{-\mu_{j}, \mu_{j}}\left(\xi_{q}\right) \\
& +\epsilon_{k}^{\mu_{x}} \quad P_{k-2}^{\mu_{j},-\mu_{j}}\left(\xi_{q}\right) P_{m-1}^{-\mu_{j}, \mu_{j}}\left(\xi_{q}\right) \\
& \text { - } \left.\quad \epsilon_{k_{j}}^{\mu_{j}} \epsilon_{m_{j}}^{\mu_{j}} P_{k-2}^{\mu_{j},-\mu_{j}}\left(\xi_{q}\right) P_{m-2}^{-\mu_{j}, \mu_{j}}\left(\xi_{q}\right)\right],
\end{aligned}
$$

which is also exact when $\mathcal{Q} \geq \mathcal{M}_{j}+2$, and the same argument on the symmetry of the matrix applies here. 


\section{References}

[1] M. Zayernouri, G. E. Karniadakis, Fractional Sturm-Liouville eigenproblems: theory and numerical approximations, J. Comp. Physics 47-3 (2013) 2108-2131.

[2] K. S. Miller, B. Ross, An introduction to the fractional calculus and fractional differential equations, New York, NY:John Wiley and Sons, Inc., 1993.

[3] I. Podlubny, Fractional differential equations, San Diego, CA, USA: Academic Press, 1999.

[4] B. J. West, M. Bologna, P. Grigolini, Physics of fractal operators, New York, NY: Springer Verlag., 2003.

[5] F. Mainardi, Fractional calculus and waves in linear viscoelasticity: an introduction to mathematical models, Imperial College Press, 2010.

[6] D. A. Benson, S. W. Wheatcraft, M. M. Meerschaert, Application of a fractional advection-dispersion equation, Water Resources Research 36 (6) (2000) 1403-1412.

[7] W. Chester, Resonant oscillations in closed tubes, J. Fluid Mech 18 (1) (1964) 44-64.

[8] J. J. Keller, Propagation of simple non-linear waves in gas filled tubes with friction, Zeitschrift für angewandte Mathematik und Physik ZAMP 32 (2) (1981) 170-181.

[9] N. Sugimoto, T. Kakutani, Generalized Burgers' equation for nonlinear viscoelastic waves, Wave motion 7 (5) (1985) 447-458.

[10] R. L. Magin, Fractional calculus in bioengineering, Redding, CT: Begell House Inc., 2006.

[11] J. P. Bouchaud, A. Georges, Anomalous diffusion in disordered media: statistical mechanisms, models and physical applications, Physics reports 195 (4) (1990) 127-293.

[12] R. Metzler, J. Klafter, The random walk's guide to anomalous diffusion: a fractional dynamics approach, Physics reports 339 (1) (2000) 1-77. 
[13] R. Klages, G. Radons, I. M. Sokolov, Anomalous Transport: Foundations and Applications, Wiley-VCH, 2008.

[14] B. Henry, S. Wearne, Fractional reaction-diffusion, Physica A: Statistical Mechanics and its Applications 276 (3) (2000) 448-455.

[15] N. Sugimoto, Burgers equation with a fractional derivative; hereditary effects on nonlinear acoustic waves, J. Fluid Mech 225 (631-653) (1991) 4 .

[16] G. Autuori, P. Pucci, Elliptic problems involving the fractional Laplacian in RN, Journal of Differential Equations 255 (8) (2013) 2340-2362.

[17] B. Gustafsson, H. O. Kreiss, J. Oliger, Time dependent problems and difference methods, vol. 67, Wiley New York, 1995.

[18] J. S. Hesthaven, S. Gottlieb, D. Gottlieb, Spectral methods for timedependent problems, vol. 21, Cambridge University Press, 2007.

[19] O. C. Zienkiewicz, R. L. Taylor, J. Z. Zhu, The finite element method: Its basis and fundamentals, Butterworth-Heinemann, 2005.

[20] G. E. Karniadakis, S. J. Sherwin, Spectral/hp element methods for CFD, Oxford University Press (2nd edition), 2005.

[21] C. Lubich, On the stability of linear multistep methods for Volterra convolution equations, IMA Journal of Numerical Analysis 3 (4) (1983) 439-465.

[22] C. Lubich, Discretized fractional calculus, SIAM Journal on Mathematical Analysis 17 (3) (1986) 704-719.

[23] J. M. Sanz-Serna, A numerical method for a partial integro-differential equation, SIAM journal on numerical analysis 25 (2) (1988) 319-327.

[24] T. Langlands, B. Henry, The accuracy and stability of an implicit solution method for the fractional diffusion equation, J. Comp. Physics 205 (2) (2005) 719-736.

[25] Z. Sun, X. Wu, A fully discrete difference scheme for a diffusion-wave system, Applied Numerical Mathematics 56 (2) (2006) 193-209. 
[26] Y. Lin, C. Xu, Finite difference/spectral approximations for the timefractional diffusion equation, Journal of Computational Physics 225 (2) (2007) 1533-1552.

[27] J. Cao, C. Xu, A high order schema for the numerical solution of the fractional ordinary differential equations, Journal of Computational Physics 238 (1) (2013) 154-168.

[28] L. Blank, Numerical treatment of differential equations of fractional order, Citeseer, 1996.

[29] E. A. Rawashdeh, Numerical solution of fractional integro-differential equations by collocation method, Applied mathematics and computation 176 (1) (2006) 1-6.

[30] X. Li, C. Xu, A space-time spectral method for the time fractional diffusion equation, SIAM Journal on Numerical Analysis 47 (3) (2009) $2108-2131$.

[31] X. Li, C. Xu, Existence and uniqueness of the weak solution of the spacetime fractional diffusion equation and a spectral method approximation, Communications in Computational Physics 8 (5) (2010) 1016.

[32] G. J. Fix, J. P. Roop, Least squares finite-element solution of a fractional order two-point boundary value problem, Computers \& Mathematics with Applications 48 (7) (2004) 1017-1033.

[33] M. M. Khader, On the numerical solutions for the fractional diffusion equation, Communications in Nonlinear Science and Numerical Simulation 16 (6) (2011) 2535-2542.

[34] C. Piret, E. Hanert, A radial basis functions method for fractional diffusion equations, J. Comp. Physics (2012) 71-81.

[35] E. Doha, A. Bhrawy, S. Ezz-Eldien, A Chebyshev spectral method based on operational matrix for initial and boundary value problems of fractional order, Computers \& Mathematics with Applications 62 (5) (2011) 2364-2373.

[36] A. H. Bhrawy, M. M. Al-Shomrani, A shifted Legendre spectral method for fractional-order multi-point boundary value problems, Advances in Difference Equations 2012 (1) (2012) 1-19. 
[37] M. Maleki, I. Hashim, M. T. Kajani, S. Abbasbandy, An adaptive pseudospectral method for fractional order boundary value problems, in: Abstract and Applied Analysis, vol. 2012, Hindawi Publishing Corporation, 2012.

[38] D. Baleanu, A. Bhrawy, T. Taha, Two efficient generalized Laguerre spectral algorithms for fractional initial value problems, in: Abstract and Applied Analysis, vol. 2013, Hindawi Publishing Corporation, 2013.

[39] A. Bhrawy, M. Alghamdia, A New Legendre spectral Galerkin and pseudo-spectral approximations for fractional initial value problems 2013.

[40] Q. Xu, J. Hesthaven, Stable multi-domain spectral penalty methods for fractional partial differential equations, Journal of Computational Physics 257 (2014) 241-258.

[41] M. Zayernouri, G. E. Karniadakis, Exponentially accurate spectral and spectral element methods for fractional ODEs, J. Comp. Physics 257 (2014) 460-480.

[42] M. Zayernouri, W. Cao, Z. Zhang, G. E. Karniadakis, Spectral and Discontinuous Spectral Element Methods for Fractional Delay Equations, SIAM Journal on Scientific Computing, (2014)-Accepted .

[43] M. Zayernouri, G. E. Karniadakis, Discontinuous Spectral Element Methods for Time- and Space-Fractional Advection Equations, SIAM Journal on Scientific Computing 36 (4) (2014) B684-B707.

[44] M. Zayernouri, G. E. Karniadakis, Fractional Spectral Collocation Method, SIAM Journal on Scientific Computing 36 (1) (2014) A40-A62.

[45] M. Zayernouri, G. E. Karniadakis, Fractional Spectral Collocation Methods for Linear and Nonlinear Variable Order FPDEs, J. Comp. Physics, A Special Issue on FPDEs, (2014)-Accepted .

[46] J. P. Roop, Variational solution of the fractional advection-dispersion equation, PhD Dissertation, Clemson University, Department of Mathematical Sciences, 2004. 
[47] J. P. Roop, Computational aspects of FEM approximation of fractional advection dispersion equations on bounded domains in R2, Journal of Computational and Applied Mathematics 193 (1) (2006) 243-268.

[48] A. Ern, Theory and practice of finite elements, vol. 159, Springer, 2004.

[49] G. E. Karniadakis, R. M. Kirby II, Parallel scientific computing in C++ and MPI: a seamless approach to parallel algorithms and their implementation, Cambridge University Press, 2003. 